\title{
Large elastoplasticity under static megabar pressures: formulation and application to compression of samples in diamond anvil cells
}

\author{
Biao Feng, ${ }^{1}$ Valery I. Levitas, ${ }^{2, *}$ and Russell J. Hemley ${ }^{3}$
}

1) Department of Aerospace Engineering, Iowa State University, Ames, Iowa 50011, USA

2) Departments of Aerospace Engineering, Mechanical Engineering, and Material Science and Engineering, Iowa State University, Ames, Iowa 50011, USA

3) Geophysical Laboratory, Carnegie Institution of Washington, Washington, D.C. 20015, USA

In high pressure research, static megabar pressures are typically produced by compression of a thin sample by two diamonds in various types of diamond anvil cells. This process is accompanied by large plastic deformation (sample thickness is reduced by a factor of 30), and finite elastic deformation of a sample and even the diamond. A thermodynamically consistent system of equations for large elastic and plastic deformation of an isotropic material obeying nonlinear elasticity and pressure dependent yield condition is formulated. The Murnaghan elasticity law and pressure-dependent $J_{2}$ plasticity are utilized. The finite-strain third-order elasticity law for cubic crystals is utilized for diamond. A computational algorithm is presented with emphasis on the stress update procedure and derivation of the consistent tangent moduli. It is implemented as a user material subroutine in the finite element code ABAQUS. Material parameters for a rhenium sample, as an example, and a diamond are calibrated based on the experimental and atomistic simulation results in the literature. The evolution of the stress and strain tensor fields in the sample and diamond is studied up to a pressure of $300 \mathrm{GPa}$. Good correspondence between numerical and experimental pressure distributions at the diamond-sample contact surface is obtained. Because there is a significant scatter of the magnitude of reported third-order single-crystal elastic moduli for diamond, their effect on strains and stresses is studied in detail. With the smaller third-order elastic moduli, the phenomenon of cupping of the diamond-sample contact surface is reproduced, which plays an important role in increasing maximum pressures for a given anvil geometry. The results provide important insight into the mechanical response in diamond anvil cells, interpretation of materials properties under extreme conditions from heterogeneous fields, and

\footnotetext{
${ }^{*}$ Corresponding author.

Email: vlevitas@iastate.edu
} 
optimum design of cells for reaching the maximum static pressure in a volume sufficient for the desired measurements.

Keywords: Large elastic and plastic deformations, Static megabar pressures, Diamond anvil cells, Computational algorithm, Finite element simulations

\section{Introduction}

The behavior of materials under high pressures is of great fundamental and applied interest. Fundamental aspects concern the search of new materials, phenomena, and unique properties that appear under extreme conditions. The interior of each planet is under high pressure; thus, geophysical research is heavily based on the study and interpretation of a material mechanical, physical, and chemical behavior under high pressure. Applied aspects include transforming highpressure discoveries in technology, e.g., industrial synthesis of diamond and cubic boron nitride (Novikov, 2005).

From the mechanical point of view, the main questions are: how it is possible to produce pressures exceeding by two orders of magnitude the yield strength of the sample and more than an order of magnitude the ultimate strength of the tool (anvils) used to produce high pressures. Both issues can be addressed by considering plastic compression with large strains of a thin sample between two anvils with a conical part. Plastic compression of a sample in the presence of friction shear stresses at the contact surface between the sample and anvils corresponds to the classical Prandtl problem (Hill, 1950) on compression of a perfectly plastic thin layer or its approximate axisymmetric solution, which is broadly used in metal forming (Eremets, 1996; Hill, 1950; Levitas, 1996; Thomsen et al., 1965). It is described by the simplified equilibrium equation and its solution

$$
\frac{d p}{d r}=-2 \tau_{f} / h ; \tau_{f}=\tau_{y} \approx 0.5 \sigma_{y} \rightarrow p=p_{R}+\sigma_{y}(R-r) / h
$$

where $p$ and $p_{R}$ is the pressure at radii $r$ and $R$, respectively, $h$ is the thickness of the thin layer, and $\tau_{f}$ is the shear friction stress at the contact surface. For a thin sample (large $R / h$ ), friction stress $\tau_{f}$ in the major part of the contact surface reaches its maximum value when it is equal to the yield strength in shear $\tau_{y}$, which is taken into account in Eq.(1). For small sample thickness $h$ and large yield strength in compression $\sigma_{y} \approx 2 \tau_{y}$, a very large pressure gradient develops. Thus, the pressure at the center exceeds pressure at the edge of a sample $R, p_{R}$, by $\sigma_{y} R / h$, i.e., for large 
$R / h$ it can be large as well. A large pressure gradient over the relatively small working area of an anvil along with the conical shape of an anvil (the principle of massive support as developed by Bridgman, 1952) allows an anvil to carry such high stresses in a small volume surrounded by a less stressed volume. Of course, the larger the yield strength of a sample (or gasket, in a hole of which the sample is placed), the higher the pressure can be achieved; the larger the strength of an anvil, the higher the pressure can be produced without fracture of an anvil.

Experiments for studying the material properties and transformations under the static pressure of several megabars are routinely produced in various types of diamond anvil cells. The characterization of anvils under those conditions have been examined in early studies by Hemley et al. 1997, Mao and Bell 1978, and Moss et al. 1986. These studies showed that the determination of stress-strain fields in both diamond and a sample/gasket is of vital importance. For complete characterization of the properties and transformations one needs to know fields of all components of the stress, and elastic and plastic strain tensors, both in a sample and diamond. Stress and strain fields in diamond anvils are required for their optimal design, preventing fracture, and reaching maximum possible pressure. In early treatments, the pressure distribution along the contact surface between a sample and diamond was measured (Goettel et al., 1985; Hemley et al., 1997; Jeanloz et al., 1991; Levitas et al., 2006; Meade and Jeanloz, 1988; Novikov et al., 1991a; Sung et al., 1977; Vohra et al., 1988; Weir et al., 1998). This distribution combined with the measured sample thickness under the load was used to determine the pressure dependence of the yield strength in shear (Jeanloz et al., 1991; Levitas et al., 1996; Sung et al., 1977; Weir et al., 1998; Zhao and Zhang, 2007), which in the major part of the contact surface is equal to the shear friction stress. Other studies in more recent works included obtaining experimental constraints on the components of the elastic strain tensor that have been measured in some selected regions of a sample using axial or radial X-ray diffraction (Duffy et al., 1999b; Hemley et al., 1997; Hemley et al., 2005; Merkel et al., 2013; Nisr et al., 2014; Singh, 1993; Singh et al., 2012; Singh et al., 1998; Wenk et al., 2007). If the pressure-dependence of the elastic moduli of a single crystal is known, stresses can be calculated. For polycrystalline samples, additional assumptions that allow connecting single and polycrystalline elastic moduli (e.g. Hashin-Shtrikman) are required for proper treatments. In this respect, methods have been developed to measure the distribution of elastic lattice strains that are used to obtain distribution of stresses (Merkel et al., 2013; Nisr et al., 2014; Wenk et al., 2007). 
Numerical methods for determination of stress and strain fields have also been developed and applied to modelling different high pressure devices. Solutions for the stress state of perfectly plastic material in different high pressure apparatuses (including Bridgman anvils) with different geometries have been obtained using slip-line methods. These devices include those employing Bridgman or diamond anvils, recessed anvils used for diamond synthesis, toroidal types of anvils, and belt apparatuses (see Levitas, 1981, 1996) and textbook by Eremets (1996). Strain state and plastic flow have not been considered in these methods and the anvils were in general considered to be rigid. Finite element method (FEM) simulations of the stress state of different types of anvils, including recessed anvils (Novikov et al., 1986), belt type devices (Levitas et al., 1986), and diamond anvils (Novikov et al., 1987), have been performed, where boundary conditions were taken from the slip-lines solutions. Strength and durability criteria for cemented carbide (Novikov et al., 1986; 1991b; 1991c; 1991d) and diamond anvils (Novikov et al., 1987; 1992; 1994) were suggested, including size effects, and optimization of anvils based on this criteria, has been developed. Notably, the criterion of maximum elongation orthogonal to the cleavage plane was found to be justified for diamond for relatively low pressure (Voronin et al., 1984). Other FEM approaches (Adams and Shaw, 1982, Bruno and Dunn, 1984) have studied stress distributions resulting from various beveled angles, where boundary conditions were schematized rather than strictly determined, and both metal gasket and diamond were generally treated as isotropic elastic materials.

Attempts to model the plastic flow in different high pressure devices using FEM simulations have been performed by Idesman and Levitas (1995), Levitas et al. (1996), Merkel et al. (1999), Moss and Goettel (1987), Moss et al. (1986), Novikov and Levitas (1985), Novikov et al. (1990). Merkel et al. (1999), Moss and Goettel (1987), and Moss et al. (1986) used the FEM code NIKE2D to simulate the behavior of the diamond anvils as well as plastic flow of a sample. Moss et al. (1986) investigated the effect of the yield strength of a gasket on the maximum achievable pressure, and found that increasing yield strength allows one to achieve higher pressure. Further, by using the "Supreme 63" tool metal with the yield strength of 2.5 GPa for a gasket, Moss et al. claimed to achieve pressures of $460 \mathrm{GPa}$ experimentally. Moss and Goettel (1987) numerically studied deformation of a sample and diamond anvils by using double beveling diamond and discussed the design of a diamond anvil by changing culet geometries. Merkel et al. $(1999,2000 a)$ extended previous FEM calculations to analyze x-ray diffraction observations at 
pressures up to $285 \mathrm{GPa}$ (Hemley et al., 1997); in particular, they included the pressure dependence of the elastic moduli of diamond. We will discuss below the FEM results in Merkel et al. (1999) and the experimental pressure distribution obtained at the contact surface in Hemley et al. (1997). Moss et al. (1986) obtained results consistent with experiments when maximum pressure was $100 \mathrm{GPa}$.

In Idesman and Levitas (1995), Levitas et al. (1996), and Novikov et al. (1990) equations and simulation results were presented for large plastic and volumetric elastic strains, but small elastic deviatoric strains in a sample. Meanwhile studies by Idesman and Levitas (1995), Novikov et al. (1990, 1991a) were devoted to processes in high pressure devices with recessed anvils for diamond synthesis, including thermoplastic effects and phase transformation from graphite to diamond in Novikov et al. (1991a). The behavior of samples in diamond anvil cells up to $50 \mathrm{GPa}$ was examined by Levitas et al. (1996). Complementing these studies is research on compression and torsion of a sample in a rotational diamond cell by Levitas and Zarechnyy (2010c). Coupled plastic flows and strain-induced phase transformations in samples in both traditional and rotational diamond anvil cells were modelled and simulated by Feng and Levitas (2013), Feng et al. (2014), Levitas and Zarechnyy (2010a, 2010b), with the diamond anvils considered as a rigid body and small elastic and transformational strains. In addition, we point out that recently static pressures above 500 GPa have been reported (Dubrovinsky et al., 2012 and 2015) using micro-semi-balls made of nanocrystalline diamond as second-stage anvils in conventional diamond anvil cells. Our focus here is on the single-crystal diamond as the anvil material using conventional megabar designs.

The main focus of this paper is to present a model, numerical algorithm, and results of simulations for pressure conditions of $\sim 300 \mathrm{GPa}$ and higher in samples compressed in classes of diamond anvil cells, when the plastic and elastic strains of the sample are large, and even elastic strains of a diamond anvil are finite. Both geometrically and physically nonlinear elasticity rules are used for elastic strains. In Section 2 the general thermodynamically consistent system of equations for large elastic and plastic deformations is presented and analyzed. Section 3 develops specific models for a sample and diamond, including an isotropic model with the Murnaghan elasticity law and pressure-dependent $\mathrm{J}_{2}$ plasticity for the sample. A finite-strain third-order elasticity law for cubic crystals is formulated for diamond. Algorithmic aspects are discussed in Section 4 , including the stress update procedure and derivation of the consistent tangent moduli 
for a sample and diamond. They are implemented as a user material subroutine in the FEM code ABAQUS. Section 5 considers the example of a rhenium sample and diamond calibrated based on the experimental and atomistic simulation results, and the evolution of the stress and strain tensor fields in a sample is discussed in Section 6. Good correspondence between numerical and experimental pressure distributions at the diamond-sample contact surface is obtained. Because there is a significant scatter of the magnitude of the third-order elastic constants for diamond in literature, their effect on strains and stresses is studied in detail. The phenomenon of cupping of the diamond-sample contact surface is reproduced and interpreted. Stress and strain fields in the diamond anvil for different third-order elastic properties are studied in Section 7. Section 8 contains concluding remarks.

\section{General equations}

At the outset, we define the notations. We designate contractions of the second-order

tensors $\boldsymbol{A}=\left\{A_{i j}\right\}$ and $\boldsymbol{B}=\left\{B_{i j}\right\}$ over one and two indices as $\boldsymbol{A} \cdot \boldsymbol{B}=\left\{A_{i j} B_{j k}\right\}$ and $\boldsymbol{A}: \boldsymbol{B}=\left\{A_{i j} B_{j i}\right\}$, respectively. Similarly for the forth-order tensor $\boldsymbol{A}$ and $\boldsymbol{B}$, contractions over one and two indices are defined as $\boldsymbol{A} \cdot \boldsymbol{B}=\left\{A_{i j k m} B_{m n l q}\right\}$ and $\boldsymbol{A}: \boldsymbol{B}=\left\{A_{i j k m} B_{m k l q}\right\}$. The subscript $s$ means symmetrization, the superscripts $t$ and -1 designate the transposition and inverse of a tensor, the subscripts $e$ and $p$ mean elastic, and plastic deformation gradient or strains, and $\boldsymbol{I}$ is the second-order unit tensor.

\subsection{Kinematics}

The motion of material with large elastic and plastic deformations is described by a vector function $\boldsymbol{r}=\boldsymbol{r}\left(\boldsymbol{r}_{0}, t\right)$, where $\boldsymbol{r}$ and $\boldsymbol{r}_{0}$ are the position vectors of material points in the actual (deformed) configuration $\Omega$ at time instant $t$ and in the reference (undeformed) configuration $\Omega_{0}$ at the instant $t_{0}$. A multiplicative decomposition of the deformation gradient

$$
\begin{aligned}
& \boldsymbol{F}=\frac{\partial \boldsymbol{r}}{\partial \boldsymbol{r}_{0}}=\boldsymbol{F}_{e} \cdot \boldsymbol{F}_{p}=\boldsymbol{R}_{e} \cdot \boldsymbol{U}_{e} \cdot \boldsymbol{R}_{p} \cdot \boldsymbol{U}_{p}=\boldsymbol{R}_{e} \cdot \boldsymbol{U}_{e} \cdot \boldsymbol{U}_{p}=\boldsymbol{V}_{e} \cdot \boldsymbol{R}_{e} \cdot \boldsymbol{U}_{p}=\boldsymbol{V}_{e} \cdot \overline{\boldsymbol{F}}_{p} \\
& \boldsymbol{R}_{p}=\boldsymbol{I} ; \quad \boldsymbol{V}_{e}=\boldsymbol{R}_{e} \cdot \boldsymbol{U}_{e} \cdot \boldsymbol{R}_{e}^{t} ; \quad \overline{\boldsymbol{F}}_{p}=\boldsymbol{R}_{e} \cdot \boldsymbol{U}_{p}
\end{aligned}
$$

into elastic $\boldsymbol{F}_{e}$ and plastic $\boldsymbol{F}_{p}$ contributions is accepted, where $\boldsymbol{F}_{p}$ is the deformation gradient obtained after a complete release of stresses in the local vicinity of each material point, $\boldsymbol{U}_{e}$ and $\boldsymbol{U}_{p}$ are symmetric elastic and plastic right stretch tensors, $\boldsymbol{V}_{e}$ is the elastic left stretch tensor, and 
$\boldsymbol{R}_{e}$ and $\boldsymbol{R}_{p}$ are the proper orthogonal elastic and plastic rotation tensors. The locally stress-free configuration is called the intermediate configuration $\Omega_{p}$. By considering motion with respect to the frame of reference rotating with the tensor $\boldsymbol{R}_{p}^{t}$ in the configuration $\Omega_{p}$, we eliminate plastic rotation and obtain $\boldsymbol{R}_{p}=\boldsymbol{I}$ and the rest of Eq. (2). This allows us to eliminate the problem of objectivity of rate equations with respect to superposition of the rigid-body rotations in $\Omega_{p}$. Still, for algorithmic purposes, it is convenient to introduce a "new" plastic deformation gradient $\overline{\boldsymbol{F}}_{p}=\boldsymbol{R}_{e} \cdot \boldsymbol{U}_{p}$. We utilized decomposition of the velocity gradient $\boldsymbol{l}=\dot{\boldsymbol{F}} \cdot \boldsymbol{F}^{-1}=\boldsymbol{W}+\boldsymbol{d}$ into symmetric deformation rate $\boldsymbol{d}=(\boldsymbol{l})_{s}$ and skew-symmetric spin $\boldsymbol{W}=(\boldsymbol{l})_{a}$ as well as $\boldsymbol{l}$ and $\boldsymbol{d}$ into elastic and dissipative contributions

$$
\boldsymbol{l}=\dot{\boldsymbol{F}}_{e} \cdot \boldsymbol{F}_{e}^{-1}+\boldsymbol{F}_{e} \cdot \dot{\boldsymbol{U}}_{p} \cdot \dot{\boldsymbol{U}}_{p}^{-1} \cdot \boldsymbol{F}_{e}^{-1}=\boldsymbol{l}_{e}+\boldsymbol{l}_{D} ; \quad \boldsymbol{d}=\left(\dot{\boldsymbol{F}}_{e} \cdot \boldsymbol{F}_{e}^{-1}\right)_{s}+\left(\boldsymbol{F}_{e} \cdot \dot{\boldsymbol{U}}_{p} \cdot \boldsymbol{U}_{p}^{-1} \cdot \boldsymbol{F}_{e}^{-1}\right)_{s}=\boldsymbol{d}_{e}+\boldsymbol{d}_{D} .
$$

As usual, we introduce the Lagrangian and Eulerian elastic strain tensor

$$
\begin{array}{r}
\boldsymbol{E}_{e}=0.5\left(\boldsymbol{F}_{e}^{t} \cdot \boldsymbol{F}_{e}-\boldsymbol{I}\right)=0.5\left(\boldsymbol{U}_{e} \cdot \boldsymbol{U}_{e}-\boldsymbol{I}\right) ; \\
\boldsymbol{B}_{e}=0.5\left(\boldsymbol{F}_{e} \cdot \boldsymbol{F}_{e}^{t}-\boldsymbol{I}\right)=0.5\left(\boldsymbol{V}_{e} \cdot \boldsymbol{V}_{e}-\boldsymbol{I}\right)=\boldsymbol{R}_{e} \cdot \boldsymbol{E}_{e} \cdot \boldsymbol{R}_{e}^{t} .
\end{array}
$$

Decomposition (3) and its further elaboration are important for derivation of the constitutive equations for anisotropic elastoplastic materials (Levitas, 1996), which involve the antisymmetric elastic spin tensor $\boldsymbol{\Omega}_{e}=\dot{\boldsymbol{R}}_{e} \cdot \boldsymbol{R}_{e}^{T}=-\boldsymbol{\Omega}_{e}^{T}$ and associated co-rotational time derivative. For isotropic materials considered here, the following kinematic decomposition will be utilized, which can be obtained from Eq. (3) (Levitas, 1996)

$$
\boldsymbol{d}=\stackrel{\nabla}{\boldsymbol{B}_{e}}-2\left(\boldsymbol{d} \cdot \boldsymbol{B}_{e}\right)_{s}+\boldsymbol{V}_{e} \cdot \boldsymbol{D}_{p} \cdot \boldsymbol{V}_{e} ; \quad \boldsymbol{D}_{p}=\boldsymbol{R}_{e} \cdot\left(\dot{\boldsymbol{U}}_{p} \cdot \boldsymbol{U}_{p}^{-1}\right)_{s} \cdot \boldsymbol{R}_{e}^{t} ; \quad \stackrel{\nabla}{\boldsymbol{B}}_{e}=\dot{\boldsymbol{B}}_{e}-2\left(\boldsymbol{W} \cdot \boldsymbol{B}_{e}\right)_{s},
$$

where $\boldsymbol{D}_{p}$ is the plastic deformation rate and $\stackrel{\nabla}{\boldsymbol{B}}$ is the Jaumann objective time derivative. We decompose (Levitas, 1996)

$$
\boldsymbol{D}_{p}=\dot{\varepsilon}_{0} \boldsymbol{I}+\boldsymbol{\gamma} ; \quad \varepsilon_{0}=1 / 3 \ln \operatorname{det} \boldsymbol{U}_{p} ; \quad \boldsymbol{I}: \boldsymbol{\gamma}=0
$$

into spherical $\dot{\varepsilon}_{0} \boldsymbol{I}$ (volumetric) and deviatoric $\gamma$ components. For a plastically incompressible material, which will be considered here, $\dot{\varepsilon}_{0}=0$. The accumulated plastic strain $q$ is determined from the equation $\dot{q}=(2 \gamma: \gamma / 3)^{0.5}$. 


\subsection{Constitutive relationships}

The second law of thermodynamics for each point is accepted as the Planck inequality (e.g., Lurie, 1990; Levitas, 1996; Clayton, 2011)

$$
D=\boldsymbol{\sigma}: \boldsymbol{d}-J^{-1} \dot{\Psi} \geq 0 ; \quad J=\rho_{0} / \rho=\operatorname{det} \boldsymbol{F} .
$$

Here $D$ is the rate of dissipation per unit deformed volume, $\sigma$ is the Cauchy stress, $\rho$ and $\rho_{0}$ are the mass densities in the actual and undeformed configurations, respectively, and $\Psi$ is the specific Helmholtz free energy per unit undeformed volume. Using Eq. (5) we evaluate:

$$
\begin{gathered}
\boldsymbol{\sigma}: \boldsymbol{d}=\boldsymbol{\sigma}:{\stackrel{\nabla}{\boldsymbol{B}_{e}}}_{e}-2 \boldsymbol{\sigma}:\left(\boldsymbol{d} \cdot \boldsymbol{B}_{e}\right)+\boldsymbol{\sigma}: \boldsymbol{V}_{e} \cdot \boldsymbol{D}_{p} \cdot \boldsymbol{V}_{e}=\boldsymbol{\sigma}:{\stackrel{\nabla}{\boldsymbol{B}_{e}}}_{e}-2 \boldsymbol{B}_{e} \cdot \boldsymbol{\sigma}: \boldsymbol{d}+\boldsymbol{V}_{e}^{2} \cdot \boldsymbol{\sigma}: \boldsymbol{D}_{p} \rightarrow \\
\left(\boldsymbol{I}+2 \boldsymbol{B}_{e}\right) \cdot \boldsymbol{\sigma}: \boldsymbol{d}=\boldsymbol{\sigma}: \stackrel{\nabla}{\boldsymbol{B}}_{e}+\boldsymbol{V}_{e}^{2} \cdot \boldsymbol{\sigma}: \boldsymbol{D}_{p} \rightarrow \boldsymbol{\sigma}: \boldsymbol{d}=\boldsymbol{V}_{e}^{-2} \cdot \boldsymbol{\sigma}: \boldsymbol{B}_{e}+\boldsymbol{\sigma}: \boldsymbol{D}_{p} .
\end{gathered}
$$

We take into account that for isotropic materials, tensors $\sigma, \boldsymbol{V}_{e}, \boldsymbol{B}_{e}$, and $\boldsymbol{D}_{p}$ are coaxial and can be permuted in the product of tensors, symmetry of $\boldsymbol{\sigma}$, as well as that $\boldsymbol{I}+\mathbf{2} \boldsymbol{B}_{e}=\boldsymbol{V}_{e}^{2}$. Finally, we simplify Eq. (8)

$$
\boldsymbol{\sigma}: \boldsymbol{d}=\boldsymbol{V}_{e}^{-2} \cdot \boldsymbol{\sigma}: \dot{\boldsymbol{B}}_{e}+\boldsymbol{\sigma}: \boldsymbol{D}_{p}
$$

because $\boldsymbol{\sigma}: \stackrel{\nabla}{\boldsymbol{B}}_{e}=\boldsymbol{\sigma}:\left(\dot{\boldsymbol{B}}_{e}-2\left(\boldsymbol{W} \cdot \boldsymbol{B}_{e}\right)_{s}\right)$ and $\boldsymbol{\sigma}:\left(\boldsymbol{W} \cdot \boldsymbol{B}_{e}\right)_{s}=\boldsymbol{\sigma}: \boldsymbol{W} \cdot \boldsymbol{B}_{e}=\boldsymbol{B}_{e} \cdot \boldsymbol{\sigma}: \boldsymbol{W}=0$ due to symmetry and coaxiality of $\boldsymbol{\sigma}$ and $\boldsymbol{B}_{e}$, and, consequently, symmetry of $\boldsymbol{B}_{e} \cdot \boldsymbol{\sigma}$.

Let us consider the simplest isotropic free energy $\Psi=\Psi\left(\boldsymbol{E}_{e}\right)=\Psi\left(\boldsymbol{B}_{e}\right)$, and neglect its dependence on plastic-strain related parameters. Substituting $\Psi\left(\boldsymbol{B}_{e}\right)$ and Eq. (9) into Eq. (7), we have

$$
D=\boldsymbol{V}_{e}^{-2} \cdot \boldsymbol{\sigma}: \dot{\boldsymbol{B}}_{e}+\boldsymbol{\sigma}: \boldsymbol{D}_{p}-J^{-1} \frac{\partial \Psi}{\partial \boldsymbol{B}_{e}}: \dot{\boldsymbol{B}}_{e}=\left(\boldsymbol{V}_{e}^{-2} \cdot \boldsymbol{\sigma}-J^{-1} \frac{\partial \Psi}{\partial \boldsymbol{B}_{e}}\right): \dot{\boldsymbol{B}}_{e}+\boldsymbol{s}: \boldsymbol{\gamma} \geq 0
$$

In Eq. (10), we used decomposition of the Cauchy stress $\sigma=-p I+s$ into deviatoric part $s$ and hydrostatic pressure $p=-\sigma: I / 3$, and take into account plastic incompressibility. Assuming that the dissipation rate is independent of $\dot{\boldsymbol{B}}_{e}$, we obtain the nonlinear elastic rule and residual dissipative inequality

$$
\boldsymbol{\sigma}=J^{-1} \boldsymbol{V}_{e}^{2} \cdot \frac{\partial \Psi}{\partial \boldsymbol{B}_{e}}=J^{-1}\left(2 \boldsymbol{B}_{e}+\boldsymbol{I}\right) \cdot \frac{\partial \Psi}{\partial \boldsymbol{B}_{e}} ; \quad D=\boldsymbol{s}: \boldsymbol{\gamma} \geq 0
$$


In order to satisfy the inequality (11), the stress $s$ should be a function of $\gamma$, otherwise one can choose $s$ and $\gamma$ in a way that $s: \gamma<0$. For the rate-independent plasticity, stress $s$ is a homogeneous degree zero function of $\gamma$, i.e.,

$$
\boldsymbol{s}=\boldsymbol{s}(\gamma, p, q)=\boldsymbol{s}(\boldsymbol{n}, p, q) ; \quad \boldsymbol{n}=\gamma /|\gamma|, \text { and }|\gamma|=(\gamma: \gamma)^{0.5}
$$

where $\boldsymbol{n}$ is the direction of the dissipative part of the deformation rate, $|\boldsymbol{n}|=1$. We include accumulated plastic strain $q$ in Eq. (12) as the only hardening variable and take into account its pressure dependence. Inverting this function, $\boldsymbol{n}=\boldsymbol{n}(\boldsymbol{s}, p, q)$, we obtain the identity

$$
\varphi(s, p, q)=\boldsymbol{n}(\boldsymbol{s}, p, q): \boldsymbol{n}(\boldsymbol{s}, p, q)-1=0 .
$$

Since for any nonzero plastic deformation rate $\gamma$, deviatoric stress belongs to the evolving surface (13) in the stress space, surface $\varphi(s, p, q)=0$ is nothing but the yield surface. If $|\gamma|=0$, the tensor $\boldsymbol{n}$ and function $\boldsymbol{s}(\boldsymbol{\gamma}, p, q)$ are undetermined. As typically done, we assume that in this case, all vectors $s$ are inside the yield surface, i.e., $\varphi(s, p, q) \leq 0$. The plastic flow rule is

$$
\gamma=|\gamma| \boldsymbol{n}(\boldsymbol{s}, p, q)
$$

Since all involved tensors $\left(\boldsymbol{\sigma}, \boldsymbol{s}, \boldsymbol{d}, \boldsymbol{B}_{e}, \stackrel{\nabla}{\boldsymbol{B}}_{e}\right.$, and $\left.\gamma\right)$ transform as objective (indifferent) ones under rigid-body rotations in the actual $\Omega$ configuration, and rotations in the intermediate $\Omega_{p}$ configuration are absent, all the above equations are objective.

\section{Specific models}

\subsection{Plastic model}

The simplest pressure-dependent $\mathrm{J}_{2}$ flow theory is used (e.g., Levitas, 1996):

$$
n=\sqrt{3 / 2} s / \sigma_{y} ; \quad \varphi=\sqrt{3 / 2 s: s}-\sigma_{y}(p, q)=0,
$$

where $\sigma_{y}$ is the yield strength and $\sigma_{i}=\sqrt{3 / 2 s: s}$ is the effective stress. Then the plastic rule is

$$
\gamma=\lambda s / \sqrt{s: s}
$$

where $\lambda \geq 0$ is a scalar function determined from the consistency condition $\dot{\varphi}=0$. 
When one describes strain dependence of the yield condition, the following results should be taken into account. Levitas (1996) found for more than 60 materials belonging to different classes (e.g., metals, alloys, rocks, oxides, compacted powders) that despite the strain-induced anisotropy and history-dependence, above some level of plastic strain $q>m$ and for a deformation path without sharp changes in directions (monotonous deformation), the initially isotropic polycrystalline materials are deformed as perfectly plastic and isotropic with a strain-historyindependent limiting surface of the perfect plasticity. This statement means that: (1) the strain hardening is saturated and the defect structure and corresponding plastic properties have reached their steady state; and (2) strain-induced anisotropy does not exhibit itself at monotonous loading. The typical values of $m$ are 1 for metals and 0.4 for rocks. These results we extracted from experiments involving heterogeneous fields from ambient pressure and pressure up to $10 \mathrm{GPa}$ in Levitas (1996) and up to $32 \mathrm{GPa}$ in Novikov et al., (1999) as well as for homogeneous compression of some metals under ambient pressure (Levitas et al., 1994). These results lead to the following relation (Levitas, 1996):

$$
\begin{gathered}
\sigma_{i}=\Phi(q)(1+a(p)) ; \\
\Phi(q)=\sigma_{y 0}\left[1+2\left(\sigma_{y}^{u} / \sigma_{y 0}-1\right)(1-0.5 q / m) q / m\right] \text { for } q / m \leq 1 ; \Phi(q)=\sigma_{y}^{u} \text { for } q>m .
\end{gathered}
$$

Here $\sigma_{y 0}$ is the yield strength at $q=p=0, \sigma_{y}^{u}$ is the maximum (saturated) yield strength that is reached after $q$ exceeds some critical value $m, a(p)$ is some function of pressure. We assume a linear pressure dependence of the yield strength. Since plastic strain in our simulations is much larger than $m$, in the current simulations we used perfectly plastic model

$$
\sigma_{i}=\sigma_{y}^{u}+b p
$$

where $b$ is the material parameter. To regularize the perfectly plastic model and avoid strain localization and mesh-dependence, weak linear strain hardening is included in some simulations.

\section{Box. 1. Complete system of equations}

Deformation gradient $\boldsymbol{F}$ is decomposed into $\boldsymbol{F}_{e}$ and $\boldsymbol{F}_{p}$

$$
\boldsymbol{F}=\partial \boldsymbol{r} / \partial \boldsymbol{r}_{0}=\boldsymbol{V}_{e} \cdot \boldsymbol{R}_{e} \cdot \boldsymbol{U}_{p}=\boldsymbol{V}_{e} \cdot \overline{\boldsymbol{F}}_{p} ; \quad \overline{\boldsymbol{F}}_{p}=\boldsymbol{R}_{e} \cdot \boldsymbol{U}_{p} ; \quad \boldsymbol{B}_{e}=0.5\left(\boldsymbol{F}_{e} \cdot \boldsymbol{F}_{e}^{t}-\boldsymbol{I}\right)=0.5\left(\boldsymbol{V}_{e} \cdot \boldsymbol{V}_{e}-\boldsymbol{I}\right) .
$$

Decomposition of the deformation rate into elastic and plastic parts 


$$
\boldsymbol{d}=\stackrel{\nabla}{\boldsymbol{B}}_{e}-2\left(\boldsymbol{d} \cdot \boldsymbol{B}_{e}\right)_{s}+\boldsymbol{V}_{e} \cdot \boldsymbol{D}_{p} \cdot \boldsymbol{V}_{e} ; \quad \boldsymbol{D}_{p}=\boldsymbol{R}_{e} \cdot\left(\dot{\boldsymbol{U}}_{p} \cdot \boldsymbol{U}_{p}^{-1}\right)_{s} \cdot \boldsymbol{R}_{e}^{t}=\boldsymbol{\gamma} ; \quad \stackrel{\nabla}{\boldsymbol{B}}_{e}=\dot{\boldsymbol{B}}_{e}-2\left(\boldsymbol{W} \cdot \boldsymbol{B}_{e}\right)_{s} .
$$

Elasticity rule

$$
\boldsymbol{\sigma}=J^{-1}\left(2 \boldsymbol{B}_{e}+\boldsymbol{I}\right) \cdot \frac{\partial \Psi}{\partial \boldsymbol{B}_{e}}
$$

Yield surface

$$
\varphi(s, p, q)=\sigma_{i}-\Phi(q)(1+b p)=0 .
$$

Plastic flow rule

$$
\gamma=\lambda s / \sqrt{s: s}
$$

$\lambda=0$ in the elastic region $(\varphi(s, p, q)<0$ or $\varphi(s, p, q)=0$ but $\dot{\varphi}(s, p, q) \leq 0)$ and $\lambda$ is determined from the consistency condition $\dot{\varphi}(s, p, q)=0$ in the elastoplastic region $(\varphi(s, p, q)=0$ and $\dot{\varphi}(s, p, q)>0)$.

Accumulated plastic strain

$$
\dot{q}=(2 \gamma: \gamma / 3)^{0.5}=\sqrt{2 / 3} \lambda .
$$

Equilibrium Equations

$$
\nabla \cdot \sigma=\mathbf{0}
$$

\subsection{Nonlinear isotropic elasticity for the sample}

The most popular elastic potential for high pressure is the third-order Murnaghan potential (Murnaghan, 1951):

$$
\Psi\left(\boldsymbol{B}_{e}\right)=\frac{\lambda_{e}+2 G}{2} I_{1}^{2}-2 G I_{2}+\left(\frac{l+2 m}{3} I_{1}^{3}-2 m I_{1} I_{2}+n I_{3}\right),
$$

where $\lambda_{e}, G, m, l$, and $n$ are material parameters, and $I_{1}, I_{2}$, and $I_{3}$, are the first, second and third invariants of the strain tensor $\boldsymbol{B}_{e}$ :

$$
I_{1}=B_{e_{11}}+B_{e_{22}}+B_{e_{33}} ; I_{3}=\operatorname{det} \boldsymbol{B}_{e} ; \quad I_{2}=B_{e_{22}} B_{e_{33}}-B_{e_{23}}^{2}+B_{e_{11}} B_{e_{33}}-B_{e_{13}}^{2}+B_{e_{22}} B_{e_{11}}-B_{e_{12}}^{2} .
$$

Then the Cauchy stress is 


$$
\boldsymbol{\sigma}=J^{-1}\left(2 \boldsymbol{B}_{e}+\boldsymbol{I}\right) \cdot \frac{\partial \Psi\left(\boldsymbol{B}_{e}\right)}{\partial \boldsymbol{B}_{e}}=J^{-1}\left(2 \boldsymbol{B}_{e}+\boldsymbol{I}\right) \cdot\left(\lambda_{e} I_{1} \boldsymbol{I}+2 G \boldsymbol{B}_{e}+\left(l I_{1}^{2}-2 m I_{2}\right) \boldsymbol{I}+n \frac{\partial I_{3}}{\partial \boldsymbol{B}_{e}}+2 m I_{1} \boldsymbol{B}_{e}\right),
$$

in which $\frac{\partial I_{3}}{\partial \boldsymbol{B}_{e}}=\left(\begin{array}{lll}B_{e 22} B_{e 33}-B_{e 23} B_{e 32} & B_{e 23} B_{e 31}-B_{e 33} B_{e 21} & B_{e 21} B_{e 32}-B_{e 22} B_{e 31} \\ B_{e 23} B_{e 31}-B_{e 33} B_{e 21} & B_{e 11} B_{e 33}-B_{e 13} B_{e 31} & B_{e 12} B_{e 31}-B_{e 11} B_{e 32} \\ B_{e 21} B_{e 32}-B_{e 22} B_{e 31} & B_{e 12} B_{e 31}-B_{e 11} B_{e 32} & B_{e 11} B_{e 22}-B_{e 12} B_{e 21}\end{array}\right)$.

\subsection{Nonlinear anisotropic elasticity for single-crystal diamond}

Only three material parameters $\left(\mathrm{C}_{11}, \mathrm{C}_{22}\right.$ and $\left.\mathrm{C}_{44}\right)$ in the Hooke's law are sufficient to describe the mechanical response under small deformations. However, for finite elastic deformations, at least cubic terms in strain elastic energy are required and six third-order elastic constants should be included (Anastassakis et al., 1990; Cousins, 2003; Grimsditch et al., 1978; Lang and Gupta, 2011; Nielsen, 1986), which have not been considered before for diamond anvil cells. For fully geometrically and physically nonlinear formulation, the consistent tangent moduli are not trivial to derive and time consuming to program. Traditional elasticity rule has the form (Lurie, 1990)

$$
\boldsymbol{\sigma}=J^{-1} \boldsymbol{F} \cdot \tilde{\boldsymbol{T}}(\boldsymbol{E}) \cdot \boldsymbol{F}^{t} ; \quad \boldsymbol{T}=\boldsymbol{\sigma d e t} \boldsymbol{F}=\boldsymbol{F} \cdot \tilde{\boldsymbol{T}}(\boldsymbol{E}) \cdot \boldsymbol{F}^{t} ; \quad \tilde{\boldsymbol{T}}=\frac{\partial \Psi}{\partial \boldsymbol{E}},
$$

where $\boldsymbol{T}$ is the Kirchhoff stress, $\tilde{\boldsymbol{T}}$ is the second Piola-Kirchhoff stress, and, since there is no plastic deformation in a diamond, subscript $e$ is dropped. Under very high pressure, it is necessary to consider at least the third-order potential $\Psi$ consistent with the cubic symmetry (Nielsen, 1986):

$$
\begin{aligned}
& \Psi=0.5 c_{11}\left(\eta_{1}^{2}+\eta_{2}^{2}+\eta_{3}^{2}\right)+c_{12}\left(\eta_{1} \eta_{2}+\eta_{1} \eta_{3}+\eta_{2} \eta_{3}\right)+0.5 c_{44}\left(\eta_{4}^{2}+\eta_{5}^{2}+\eta_{6}^{2}\right)+c_{111}\left(\eta_{1}^{3}+\eta_{2}^{3}+\eta_{3}^{3}\right) / 6 \\
& +0.5 c_{112}\left[\eta_{1}^{2}\left(\eta_{2}+\eta_{3}\right)+\eta_{2}^{2}\left(\eta_{1}+\eta_{3}\right)+\eta_{3}^{2}\left(\eta_{1}+\eta_{2}\right)\right]+c_{123} \eta_{1} \eta_{2} \eta_{3}+0.5 c_{144}\left(\eta_{1} \eta_{4}^{2}+\eta_{2} \eta_{5}^{2}+\eta_{3} \eta_{6}^{2}\right) \\
& +0.5 c_{166}\left[\left(\eta_{2}+\eta_{3}\right) \eta_{4}^{2}+\left(\eta_{1}+\eta_{3}\right) \eta_{5}^{2}+\left(\eta_{1}+\eta_{2}\right) \eta_{6}^{2}\right]+c_{456} \eta_{4} \eta_{5} \eta_{6},
\end{aligned}
$$

where $\eta_{1}=E_{11}, \eta_{2}=E_{22}, \eta_{3}=E_{33}, \eta_{4}=2 E_{23}, \eta_{5}=2 E_{31}$, and $\eta_{6}=2 E_{12}$.

The following equations give two explicit examples for the components of the second PiolaKirchhoff stress: 


$$
\begin{aligned}
& \tilde{T}_{11}=\frac{\partial \Psi}{\partial \eta_{1}}=c_{11} \eta_{1}+c_{12}\left(\eta_{2}+\eta_{3}\right)+c_{111} \eta_{1}^{2} / 2+c_{112}\left[2 \eta_{1}\left(\eta_{2}+\eta_{3}\right)+\eta_{2}^{2}+\eta_{3}^{2}\right] / 2+c_{123} \eta_{2} \eta_{3} \\
& +c_{144} \eta_{4}^{2} / 2+c_{166}\left(\eta_{5}^{2}+\eta_{6}^{2}\right) / 2 ; \quad \tilde{T}_{12}=\frac{\partial \Psi}{\partial \eta_{6}}=c_{44} \eta_{6}+c_{144} \eta_{3} \eta_{6}+c_{166}\left(\eta_{1}+\eta_{2}\right) \eta_{6}+c_{456} \eta_{4} \eta_{5} .
\end{aligned}
$$

Note that, as an alternative to Lagrangian strain based elasticity theories, the finite strain theories based on Eulerian strain (Nielsen, 1986, Clayton, 2013) and logarithmic strain (Clayton, 2014) were developed and used to describe the nonlinear elasticity of cubic diamond.

\section{Numerical algorithms}

Although there is great success in the algorithmic developments for large plastic strains using FEM (Fish and Shek, 1999, Khoei and Bakhshiani, 2004, Ponthot, 2002, Voyiadjis and Abed, 2006), they are mostly focused on typical metal forming conditions, i.e., small elastic strains obeying Hooke's law and the pressure-independent yield condition. In the paper, we will focus on the stress update algorithm for nonlinear elastic constitutive equations with finite elastic and huge plastic strains and pressure-dependent yield condition. For the large deformation problems, the convergence of FEM algorithms is an essential issue, which can be improved by utilizing consistent tangent moduli. Due to multiple nonlinearities in the kinematic and constitutive equations and large rotation, it is not trivial to derive and implement them in a subroutine. In the paper, a detailed derivation of consistent tangent moduli for both diamond and sample will be introduced.

\subsection{Update of Cauchy stress for an elastoplastic material}

We formulate a radial return algorithm for our model. Let at time $t_{n}$ the current configuration and all fields be known. We need to find them at time $t_{n+1}=t_{n}+\Delta t$ for the known time increment $\Delta t$ and known deformation gradient $F_{n+1}$. We evaluate

$$
l_{n+1}=\left(F_{n+1}-F_{n}\right) \cdot F_{n+1}^{-1} / \Delta t ; \quad \boldsymbol{d}_{n+1}=l_{s} .
$$

As the usual first step (corresponding to the iteration $i=0$ ), we consider the elastic predictor, i.e., assume that there is no change in plastic deformation gradient $U_{p}$ during time increment $\Delta t$. Then

$$
F_{n+1}=F_{e}^{0} \cdot U_{p n} \rightarrow F_{e}^{0}=F_{n+1} \cdot U_{p n}^{-1}=V_{e(n+1)}^{0} \cdot R_{e(n+1)}^{0},
$$


where $F_{e}^{0}$ is the trial elastic left stretch tensor for elastic predictor. Note that in all cases, decomposition of a nonsymmetric tensor into symmetric and orthogonal parts is performed with a FORTAN subroutine. Substituting $\boldsymbol{B}_{e(n+1)}^{0}=0.5\left(\boldsymbol{F}_{e}^{0} \cdot \boldsymbol{F}_{e}^{t 0}-\boldsymbol{I}\right)$ into elasticity rule (29), we obtain the trial Cauchy stress $\sigma^{0}$ and its deviator $s^{0}$ and spherical $-p^{0} \mathbf{I}$ parts. If $\varphi\left(s^{0}, p^{0}, q_{n}\right)<0$, this completes the update of all parameters. If $\varphi\left(s^{0}, p^{0}, q_{n}\right) \geq 0$, the plastic flow rule shall be integrated within time $\Delta t$ and stress should be iteratively reduced to satisfy the yield condition $\varphi\left(\boldsymbol{s}_{n+1}, p_{n+1}, q_{n+1}\right)=0$. In this stage, $F_{n+1}^{c}$ is fixed, and consequently $\boldsymbol{d}_{\mathrm{n}+1}^{c}=\boldsymbol{W}_{\mathrm{n}+1}^{c}=\mathbf{0}$, where superscript $c$ means the plastic corrector. Then, the kinematic Eq. (21) reduces to

$$
\dot{\boldsymbol{B}}_{e}+\boldsymbol{V}_{e} \cdot \boldsymbol{D}_{p} \cdot \boldsymbol{V}_{e}=\mathbf{0} .
$$

Substituting Eq. (35) into the plastic flow rule (24), we obtain for plastic corrector stage

$$
\dot{\boldsymbol{B}}_{e}=-\lambda \boldsymbol{V}_{e} \cdot \frac{s}{\sqrt{s: s}} \cdot \boldsymbol{V}_{e} .
$$

Integrating Eq.(36) for $\boldsymbol{B}_{e}$ from iteration step $i$ to $i+1$, we derive

$$
\boldsymbol{B}_{e(\mathrm{n}+1)}^{(\mathrm{i}+1)}=\boldsymbol{B}_{e(\mathrm{n}+1)}^{(\mathrm{i})}-\lambda \boldsymbol{V}_{e(\mathrm{n}+1)}^{(\mathrm{i})} \cdot s_{\mathrm{n}+1}^{(\mathrm{i})} / \sqrt{\sqrt{s_{\mathrm{n}+1}^{(\mathrm{i})}: s_{\mathrm{n}+1}^{(\mathrm{i})}}} \cdot \boldsymbol{V}_{e(\mathrm{n}+1)}^{(\mathrm{i})} \Delta t,
$$

where $\lambda$ is a scalar to be determined. Substituting elastic strain $\boldsymbol{B}_{e(\mathrm{n}+1)}^{(\mathrm{i}+1)}$ in the elasticity rule (22), we obtain an expression for the Cauchy stress

$$
\boldsymbol{\sigma}_{n+1}^{(\mathrm{i}+1)}=\left(\operatorname{det} \boldsymbol{F}_{n+1}\right)^{-1}\left(2 \boldsymbol{B}_{e(\mathrm{n}+1)}^{(\mathrm{i}+1)}+\boldsymbol{I}\right) \frac{\partial \Psi\left(\boldsymbol{B}_{e(\mathrm{n}+1)}^{(\mathrm{i}+1)}\right)}{\partial \boldsymbol{B}_{e(\mathrm{n}+1)}^{(\mathrm{i}+1)}} .
$$

Integrating the equation for the accumulated plastic strain, we update

$$
q_{n+1}^{i+1}=q_{n+1}^{i}+\sqrt{2 / 3} \lambda \Delta t
$$

The obtained parameters should satisfy the yield condition

$$
\varphi\left(s_{n+1}^{i+1}, p_{n+1}^{i+1}, q_{n+1}^{i+1}\right)=0 .
$$

Since all arguments of the yield condition depend on a single parameter $\lambda$, this nonlinear equation was iteratively solved using a Newton-Raphson method with the help of a developed Fortran subroutine. Due to geometrical and physical nonlinearity of the elasticity rule, pressure $p_{n+1}^{i+1}$ also 
depends on $\lambda$. Thus, $\boldsymbol{\sigma}_{n+1}^{(\mathrm{i}+1)}, \boldsymbol{B}_{e(\mathrm{n}+1)}^{(\mathrm{i}+1)}$, and $q_{n+1}^{i+1}$ are updated. Tensor $\boldsymbol{V}_{e(\mathrm{n}+1)}^{(\mathrm{i}+1)}$ can be found through known $\boldsymbol{B}_{e(\mathrm{n}+1)}^{(\mathrm{i}+1)}$ either from equation $\boldsymbol{V}_{e(\mathrm{n}+1)}^{(\mathrm{i}+1)} \cdot \boldsymbol{V}_{e(\mathrm{n}+1)}^{(\mathrm{i}+1)}=2 \boldsymbol{B}_{e(\mathrm{n}+1)}^{(\mathrm{i}+1)}+\boldsymbol{I}$ in principle axes or by solving the linearized incremental version of this equation,

$$
2\left(\boldsymbol{B}_{e(\mathrm{n}+1)}^{(\mathrm{i}+1)}-\boldsymbol{B}_{e(\mathrm{n}+1)}^{(\mathrm{i})}\right)=\left(\boldsymbol{V}_{e(\mathrm{n}+1)}^{(\mathrm{i}+1)}-\boldsymbol{V}_{e(\mathrm{n}+1)}^{(\mathrm{i})}\right) \cdot V_{e(\mathrm{n}+1)}^{(\mathrm{i})}+V_{e(\mathrm{n}+1)}^{(\mathrm{i})} \cdot\left(\boldsymbol{V}_{e(\mathrm{n}+1)}^{(\mathrm{i}+1)}-\boldsymbol{V}_{e(\mathrm{n}+1)}^{(\mathrm{i})}\right) .
$$

For these purposes, FORTRAN subroutines were written. Then, the plastic deformation gradient $\overline{\boldsymbol{F}}_{p(n+1)}^{i+1}$ and its symmetric $\boldsymbol{U}_{p(n+1)}^{i+1}$ and orthogonal $\boldsymbol{R}_{\mathrm{e}(n+1)}^{i+1}$ parts are updated by

$$
\overline{\boldsymbol{F}}_{p(n+1)}^{i+1}=V_{e(n+1)}^{-1(\mathrm{i}+1)} \cdot F_{n+1}=\boldsymbol{R}_{\mathrm{e}(n+1)}^{i+1} \cdot \boldsymbol{U}_{p(n+1)}^{i+1}
$$

In fact, with a small modification of the algorithm, we do not need to find $\boldsymbol{U}_{p(n+1)}^{i+1}$ and $\boldsymbol{R}_{\mathrm{e}(n+1)}^{i+1}$ separately because it does not matter (after constitutive equations are derived) whether $\boldsymbol{R}_{\mathrm{e}(n+1)}^{i+1}$ will be combined with elastic or plastic deformation gradients. Thus, instead of Eq. (21) we use

$$
F_{n+1}=F_{e}^{0} \cdot \overline{\boldsymbol{F}}_{p n} \rightarrow F_{e}^{0}=F_{n+1} \cdot \overline{\boldsymbol{F}}_{p n}^{-1},
$$

where $\overline{\boldsymbol{F}}_{p n}$ is found in the previous step using Eq. (42) and is kept constant during elastic predictor step. We are using $F_{e}^{0}$ instead of $V_{e}^{0}$ in Eq. (43) because elastic deformation at the fixed $\overline{\boldsymbol{F}}_{p n}$ generally is accompanied by rotations and the tensor $F_{n+1} \cdot \overline{\boldsymbol{F}}_{p n}^{-1}$ is not symmetric. This does not change the rest of the algorithm. Note that equation $\boldsymbol{D}_{p}=\boldsymbol{R}_{e} \cdot\left(\dot{\boldsymbol{U}}_{p} \cdot \boldsymbol{U}_{p}^{-1}\right)_{s} \cdot \boldsymbol{R}_{e}^{t}$ is not used in calculations. The above procedure is summarized in the following flowchart.

While the above equations may give an impression that they are for an explicit time integration scheme, since the plastic multiplier $\lambda$ is determined iteratively using the radial return algorithm to satisfy the yield condition, this is an implicit scheme (Simo and Hughes, 1998). To justify that the chosen time (load) step provides a solution which is independent of the time step, we used 6000 time steps to reach the pressure $285 \mathrm{GPa}$ and obtained the same results as with 4000 and 10000 time steps. 


\subsection{Stress update for anisotropic elastic material}

The deformation gradient $F_{n+1}$ is known and $\sigma_{n+1}$ is to be defined based on $F_{n+1}$.

1. Compute the Lagrangian strain

$$
\boldsymbol{E}_{n+1}=0.5\left(\boldsymbol{F}_{n+1}^{t} \cdot \boldsymbol{F}_{n+1}-\boldsymbol{I}\right) .
$$

2. Compute the second Piola-Kirchhoff and Cauchy stresses using Eqs. (30)-(32)

$$
\tilde{\boldsymbol{T}}_{n+1}=\frac{\partial \Psi_{n+1}}{\partial \boldsymbol{E}_{n+1}} ; \quad \boldsymbol{\sigma}_{n+1}=\frac{1}{d e t \boldsymbol{F}_{n+1}} \boldsymbol{F}_{n+1} \cdot \tilde{\boldsymbol{T}}_{n+1} \cdot \boldsymbol{F}_{n+1}^{t} .
$$

\subsection{Consistent tangent moduli for the sample}

The consistent tangent moduli $\boldsymbol{C}$, which are determined from the following equation in ABAQUS 6.11 Manual (see Ref. 1)

$$
(J \boldsymbol{\sigma})^{\bullet}=J(\boldsymbol{C}: \boldsymbol{d}+\boldsymbol{W} \cdot \boldsymbol{\sigma}-\boldsymbol{\sigma} \cdot \boldsymbol{W}) ; \quad J=\operatorname{det} F
$$

shall be evaluated in the user-defined subroutine UMAT. The fourth order tensor $\boldsymbol{C}$ does not affect the accuracy of results but affects the convergence rate, especially for large elastic and plastic deformation problems. From Eq. (46), it is obtained that

$$
\boldsymbol{C}: \boldsymbol{d}=\frac{\dot{J}}{J} \boldsymbol{\sigma}+(\dot{\boldsymbol{\sigma}}+\boldsymbol{\sigma} \cdot \boldsymbol{W}-\boldsymbol{W} \cdot \boldsymbol{\sigma}) ; \quad \boldsymbol{C}=\boldsymbol{\sigma} \frac{1}{J} \frac{\partial \dot{J}}{\partial \boldsymbol{d}}+\frac{\partial \boldsymbol{\nabla}}{\partial \boldsymbol{d}}
$$

Normally, $\sigma \frac{1}{J} \frac{\partial \dot{J}}{\partial \boldsymbol{d}}$ is not considered in literature, because under low pressure $\dot{J} \approx 0$. However, without considering this term, a very severe convergence problem appears at megabar pressures and the exact definitions of both terms in right side of Eq. (47) are essential.

\subsubsection{Consistent tangent moduli for an elastoplastic material}

Since

$$
\dot{J}=J \dot{\boldsymbol{F}}: \boldsymbol{F}^{-1}=J \boldsymbol{I}: \boldsymbol{d}
$$

(see, e.g., Lurie (1990), Levitas (1996)), then

$$
\frac{\partial \dot{J}}{\partial \boldsymbol{d}}=J \boldsymbol{I} ; \boldsymbol{\sigma} \frac{1}{J} \frac{\partial \dot{J}}{\partial \boldsymbol{d}}=\boldsymbol{\sigma} I
$$


which contributes to $C_{i j k l}$ in Eq. (47) as $\sigma_{i j} \delta_{k l}$. The Cauchy stress is a function of tensor $\boldsymbol{B}_{e}$ only,

$$
\begin{aligned}
& \boldsymbol{\sigma}=\boldsymbol{f}\left(\boldsymbol{B}_{e}\right), \text { and } \\
& \stackrel{\nabla}{\boldsymbol{\sigma}}=\frac{\partial \boldsymbol{f}\left(\boldsymbol{B}_{e}\right)}{\partial \boldsymbol{B}_{e}}: \boldsymbol{B}_{e}=\frac{\partial \boldsymbol{f}\left(\boldsymbol{B}_{e}\right)}{\partial \boldsymbol{B}_{e}} \cdot\left(2 \boldsymbol{B}_{e}+\boldsymbol{I}\right):(\boldsymbol{d}-\boldsymbol{n}|\gamma|)=\boldsymbol{Y}\left(\boldsymbol{B}_{e}\right):(\boldsymbol{d}-\boldsymbol{n}|\gamma|) ; \boldsymbol{Y}\left(\boldsymbol{B}_{e}\right)=\frac{\partial \boldsymbol{f}\left(\boldsymbol{B}_{e}\right)}{\partial \boldsymbol{B}_{e}} \cdot\left(2 \boldsymbol{B}_{e}+\boldsymbol{I}\right),
\end{aligned}
$$

where Eq. (4.53) from Levitas (1996) was specified for our model. Next, it follows from the consistency condition

$\dot{\varphi}=\frac{\partial \varphi}{\partial \boldsymbol{\sigma}}: \boldsymbol{\sigma}+\frac{\partial \varphi}{\partial q} \sqrt{\frac{2}{3} \gamma: \boldsymbol{\gamma}}=\frac{\partial \varphi}{\partial \boldsymbol{\sigma}}: \boldsymbol{Y}\left(\boldsymbol{B}_{e}\right):(\boldsymbol{d}-\boldsymbol{n}|\gamma|)+\left(\frac{2}{3}\right)^{0.5} \frac{\partial \varphi}{\partial q}|\gamma|=\frac{\partial \varphi}{\partial \boldsymbol{\sigma}}: \boldsymbol{Y}\left(\boldsymbol{B}_{e}\right): \boldsymbol{d}-H|\gamma|=0$,

where $H=\left\{\frac{\partial \varphi}{\partial \boldsymbol{\sigma}}: \boldsymbol{Y}\left(\boldsymbol{B}_{e}\right): \boldsymbol{n}-(2 / 3)^{0.5} \frac{\partial \varphi}{\partial q}\right\}$

Resolving Eq. (51) for $|\gamma|$,

$$
|\gamma|=\frac{\partial \varphi}{\partial \boldsymbol{\sigma}}: \boldsymbol{Y}\left(\boldsymbol{B}_{e}\right) / H: \boldsymbol{d}
$$

and substituting Eq. (52) into Eq. (50), we obtain

$$
\nabla=\left[\boldsymbol{Y}\left(\boldsymbol{B}_{e}\right)-\boldsymbol{Y}\left(\boldsymbol{B}_{e}\right): \boldsymbol{n}\left(\frac{\partial \varphi}{\partial \boldsymbol{\sigma}}: \boldsymbol{Y}\left(\boldsymbol{B}_{e}\right) / H\right)\right]: \boldsymbol{d} .
$$

Thus, using definition Eq.(47) for the consistent tangent moduli $\boldsymbol{C}$, and Eqs. (49) and (53), we finally obtain

$$
\boldsymbol{C}=\boldsymbol{\sigma} I+\boldsymbol{Y}\left(\boldsymbol{B}_{e}\right)-\boldsymbol{Y}\left(\boldsymbol{B}_{e}\right): \boldsymbol{n}\left(\frac{\partial \varphi}{\partial \boldsymbol{\sigma}}: \boldsymbol{Y}\left(\boldsymbol{B}_{e}\right) / H\right) .
$$

For the simplified case of small elastic strains, linear elasticity, and pressure-independent yield strength, the current model coincides with the model in Levitas and Zarechnyy (2010c). Solutions of the problem on compression of a sample (similar to that solved in the current paper) and compression and torsion of a sample were found in Levitas and Zarechnyy (2010c) using standard ABAQUS code without UMAT subroutines. These solutions were compared in Levitas and Zarechnyy (2010c) with simplified analytical solutions for compression and compression and torsion and good correspondence was found. Here, we compared solutions for compression of a sample obtained with the current UMAT subroutines for the simplified model considered in 
Levitas and Zarechnyy (2010c) with the solutions using standard ABAQUS code without UMAT subroutines and found very good agreement. This gives us confidence that our algorithms and UMAT subroutines work correctly.

\subsubsection{Consistent tangent moduli for anisotropic elasticity for cubic crystal}

Since elasticity rule and Eq. (46) are simpler in terms of the Kirchhoff stress $T$ and there is no plasticity condition, which is expressed in terms of the Cauchy stress, it is easier to work in terms of the Kirchhoff stress $T$. Thus, Eq. (47) can be rewritten as

$$
\boldsymbol{C}: \boldsymbol{d}=J^{-1} \stackrel{\nabla}{\boldsymbol{T}} ; \quad \boldsymbol{C}=J^{-1} \frac{\partial \boldsymbol{\nabla}}{\partial \boldsymbol{d}}
$$

We can utilize Eq. (6.2.15) from Lubarda (2001)

$$
\stackrel{\nabla}{\boldsymbol{T}}=(\boldsymbol{L}+2 \boldsymbol{K}): \boldsymbol{d}, \text { i.e., } \boldsymbol{C}=J^{-1}(\boldsymbol{L}+2 \boldsymbol{K}),
$$

where the forth order tensors $\boldsymbol{L}$ and $\boldsymbol{K}$ are

$$
\boldsymbol{L}=\left(\boldsymbol{F} \cdot \boldsymbol{F} \cdot \frac{\partial^{2} \Psi}{\partial \boldsymbol{E} \partial \boldsymbol{E}} \cdot \boldsymbol{F}^{t} \cdot \boldsymbol{F}^{t}\right) ; \quad K_{i j k l}=\frac{1}{4}\left(T_{i k} \delta_{j l}+T_{j k} \delta_{i l}+T_{i l} \delta_{j k}+T_{j l} \delta_{i k}\right) .
$$

All parameters in Eq. (56) are either known or can be easily calculated. For example,

$$
\frac{\partial^{2} \Psi}{\partial \eta_{1} \partial \eta_{1}}=c_{11}+c_{111} \eta_{1}+c_{112}\left(\eta_{2}+\eta_{3}\right) ; \quad \frac{\partial^{2} \Psi}{\partial \eta_{1} \partial \eta_{2}}=c_{12}+c_{112}\left(\eta_{1}+\eta_{2}\right)+c_{123} \eta_{3} .
$$

\section{Material properties}

\subsection{Yield Condition for rhenium sample}

Under high pressure, the yield strength of a sample is the most important parameter, as it largely controls the stress-strain distribution in a sample. Rhenium is a heavy group-VII transition metal, which crystallizes in the hexagonal-close-packed structure. Rhenium is widely used as a gasket material in a diamond anvil cell due to its large bulk $(K)$ and shear $(G)$ moduli and high enough compressive strength (Jeanloz et al., 1991; Manghnani et al., 1974; Vohra et al., 1987) to retain appreciable thickness even at several megabar pressures without any "punch-through" at the edges of the diamond anvils. Usually, a rhenium gasket is either polycrystalline or compacted powder. The grain size at a pressure of $250 \mathrm{GPa}$ is $10-20 \mathrm{~nm}$ (Singh et al., 2012), which is 20 to 40 times smaller than the sample thickness at the same pressure. 
Despite the scatter in experimental data (Fig. 1), the following linear approximation to the yield strength in shear is obtained

$$
\tau_{y}=4.619+0.0231 p(\mathrm{GPa}),
$$

which is close to the approximation in Jeanloz et al. (1991). To transform this condition into yield strength in compression $\sigma_{y}$ in the pressure-dependent $J_{2}$ theory, we have to multiply $\tau_{y}$ by $\sqrt{3}$ :

$$
\sigma_{y}=8.00+0.04 p(\mathrm{GPa}) \text {. }
$$

\subsection{Elastic properties of rhenium}

In the literature (Lv et al., 2012, Manghnani et al., 1974), the bulk $K$ and shear $G$ moduli and their pressure derivatives at zero pressure are available from experiments and first-principle calculations: $K=380 \mathrm{GPa}, G=200 \mathrm{GPa}, \partial K / \partial p=4.6, \partial G / \partial p=1.7$. Note that at $\sim 300 \mathrm{GPa}$, shear modulus increases by more than a factor of three and bulk modulus increases by more than a factor of four. Because of the lack of data, we assume $n=0$ and use equations

$$
\lambda_{e}=K-2 G / 3 \quad l=-\left(\frac{\partial K}{\partial p}\right) K / 6 \quad m=-\left(\frac{\partial G}{\partial p}\right) K / 3-\lambda_{e}-G,
$$

which connect Murnaghan's constants and second-order elastic constants and their pressure derivative (Lurie, 1990). Thus, we obtain $\lambda_{e}=247 \mathrm{GPa}, l=-291 \mathrm{GPa}$, and $m=-662 \mathrm{GPa}$.

\subsection{Elastic properties of diamond}

Three second-order elastic constants are well known in literature (e.g. (McSkimin and Andreatc., 1972; Nielsen, 1986; Novikov et al., 1994)) and the discrepancies between various sources are very small. Here, the following parameters (units are in GPa) are used: $c_{11}=1050, c_{12}=127$, and $c_{44}=550$.

For the six third-order elastic constants, there are very large discrepancies in different references (see Lang and Gupta, 2011), but all of them give very good correspondence with experiments for pressure below $100 \mathrm{GPa}$ under shock compression. In this paper, the third-order elastic constants (units are in GPa) from (Lang and Gupta, 2011) are $c_{111}=-7603, c_{112}=-1909, c_{123}=-835$, $c_{144}=1438, c_{166}=-3938, c_{456}=-2316$, and from (Nielsen, 1986) are $c_{111}=-6000, c_{112}=-700$ $, c_{123}=-400, c_{144}=-300, c_{166}=-2500, c_{456}=-1300$, will be used in simulations and results will be compared. Also, the third-order elastic constants from Lang and Gupta (2011)) multiplied by a 
factor of 0.8 will be used, which in fact provide much better (than without 0.8 ) correspondence between calculated and experimental anvil profile under pressure of $285 \mathrm{GPa}$.

\section{Evolution of the stress-strain fields in a sample}

\subsection{Problem formulation}

A scheme of a conventional megabar diamond cell is shown in Fig. 2. The diamond anvil is subjected to a homogeneous normal stress $\sigma_{n}$. The loading and geometry of a conventional diamond cell are axisymmetric typically with the 001 orientation along the $z$ axis. Although the diamond is not isotropic and has a cubic symmetry, the heterogeneity in circumferential direction is very small due to $\left(c_{11}-c_{12}\right) /\left(2 c_{44}\right) \approx 1$. In particular, in Hemley et al., 1997 , the 3D experimental image of a sample thickness is practically axisymmetric. Due to symmetry, a quarter of a pre-indented sample and anvil will be treated. The geometric parameters of an anvil and sample can be found in Figs. $2 \mathrm{~b}$ and $\mathrm{c}$.

The boundary conditions for a quarter of a structure in Fig. 2 (b) are as follow:

(1) At the top of the anvil surface, the normal stress $\sigma_{n}$ and zero shear stresses are applied.

(2) At the axis $r=0$ (the line $\mathrm{AC}$ for the anvil and the line $\mathrm{CD}$ for the sample), the radial displacement $u_{r z}$ and shear stress $\tau_{r z}$ are zero.

(3) At the contact surface, the Coulomb friction model is applied. When friction stress reaches $\mu \sigma_{c}$, slipping is allowed ( $\sigma_{c}$ is the normal contact stress at the contact surface between the diamond and the sample). Otherwise, the cohesion condition, i.e., continuity of displacements, is applied.

(4) At the symmetry plane $z=0$ (plane DH in Fig. 2(c)), the radial shear stress $\tau_{r z}=0$, and the axial displacement $u_{z}=0$.

(5) Other surfaces not mentioned above are stress-free.

Quadrilateral 4-node bilinear axisymmetric finite elements have been used in simulations, which are the most common type of elements for the large deformation model for axisymmetric problems (see, e.g., book by Dunne and Petrinic, 2005). Fig. 3 shows the mesh-independence of 
the simulation results. Our simulations utilize a mesh (Fig. 3a), with 2983 elements and the region with the larger deformation has the finer mesh. For comparison, a rough mesh with 796 elements is also shown (Fig. 3b). When the maximum pressure at the center of a sample reaches $285 \mathrm{GPa}$, results for the pressure distribution in the sample in Figs. $3 \mathrm{c}$ and d, and at the contact surface in Fig. 3e, for both fine and rough meshes are practically the same.

\subsection{Evolution of pressure and contact stresses}

The evolution of pressure with the growth of the applied normal stress $\sigma_{n}$ is shown in Fig. 4. The pressure continuously increases, and the pressure gradient is very large along the radial direction, but quite small along the thickness. The thickness of the sample reduces drastically, from the $80 \mu \mathrm{m}$ down to $2.6 \mu \mathrm{m}$. The reduction of thickness is not uniform along the radial direction, which causes the change in slope of the contact line between diamond and sample from the initial $8.5^{\circ}$ at contact region $C G$ of Fig. 2 c to the value slightly larger than zero near the point $G$.

Fig. 5a presents the comparison of pressure distributions at the contact surface between experimental and simulation results. In the experimental data the pressure gradient reduces with the growing radial coordinate, which is completely different from our previous simulation results. In Feng et al. (2013) and Levitas and Zarechnyy (2010a) the pressure gradient was zero at the center when the anvil was flat at the central part and was considered as the rigid body. In the previous FEM modeling of this experiment, the pressure gradient grows with a rising radial coordinate (Merkel et al., 1999), which is not fully consistent with the experimental results. Current results give a better correspondence than the results in Merkel et al. (1999) in Fig. 5a. When the maximum pressure is $285 \mathrm{GPa}$, the experimental and simulated distributions practically coincide. There is still some discrepancy at the periphery for the other two cases, which can be caused by differences in yield strengths at low pressure. Indeed, at the low pressure there is a very large discrepancy in experimental values of the yield strength in different papers (Duffy et al., 1999a; Jeanloz et al., 1991; Singh et al., 2012) and even in the same experiments by measurements in anvil and sample (Jeanloz et al., 1991).

Fig. 6 shows the distributions of the friction stress $\tau$, the yield strength in shear $\tau_{y}(p)$, and $\mu \sigma_{c}$. At the applied normal stress $\sigma_{n}=1.022 \mathrm{GPa}$ in Fig. 6a, the yield strength $\tau_{y}$ is larger than $\mu \sigma_{c}$ everywhere, and the friction stress $\tau$ is equal to $\mu \sigma_{c}$ in the slipping zone $(r \geq 5 \mu m)$ 
and smaller than $\mu \sigma_{c}$ in the small cohesion region with $r<5 \mu m$. It is expected that the friction stress $\tau$ near the center is small and cannot meet the Coulomb slipping condition. With the increase of the applied stress $\sigma_{n}$, the rate of increase of $\mu \sigma_{c}$ is faster than the rate of increase of yield strength in shear. Thus, $\mu \sigma_{c}>\tau_{y}$ in the central region and $\mu \sigma_{c} \leq \tau_{y}$ at the periphery. Except for the very small cohesion region at the center, shear stress at the contact surface is $\tau=\min \left(\mu \sigma_{c}, \tau_{y}(p)\right)$. With the further increase of the applied normal stress $\sigma_{n}$, the region with the Coulomb sliding at the periphery reduces and the region with $\mu \sigma_{c}>\tau_{y}$ at the center increases. In Figs. 6b, 6c and 6d, there is a very large region with $\mu \sigma_{c}>\tau_{y}$, where $\tau$ cannot reach $\mu \sigma_{c}$. Consequently, slipping at the contact surface of the diamond and sample based on the Coulomb model is not allowed there, and the cohesion condition is applied. In this case, a very large plastic shear strain along the contact surface appears in a thin layer of a sample near the contact surface (see Fig. 7). Because the direction of plastic flow is tangent to the contact surface, we call it internal plastic slipping. The sliding at the contact surfaces at $\mu \sigma_{c}>\tau_{y}$ is substituted with internal plastic slipping in this thin layer of a sample. The Coulomb friction coefficient between diamond and gasket $\mu$ is 0.1 . As it follows from Fig. 6 , in a major part of the contact surface under megabar pressures, the cohesion condition, $\mu \sigma_{c}>\tau_{y}$, is met, and the Coulomb friction coefficient is not relevant within this region. Therefore, the effect of the specific value of the friction coefficient is much smaller than the effect of the yield strength, which was also stated in (Merkel et al., 1999).

\subsection{Evolution of plastic strain and stress tensor fields}

In this paper, Fig. 7 presents the evolution of the field of the accumulated plastic strain $q$ with the growing applied normal stress $\sigma_{n}$. Initially at $\sigma_{n}=1.022 \mathrm{GPa}$, the accumulated plastic strain reduces from the center to periphery, and there is no large gradient along the thickness. For $\sigma_{n}=1.022 \mathrm{GPa}$, the Coulomb slipping condition is satisfied everywhere but in the small cohesion zone $r<5 \mu m$, the material slides at the contact surface, and thus there is no very large shear strain localization at the contact surface. For larger applied stresses, the region with plastic slipping appears at the central part of a sample and grows to the periphery. The plastic sliding condition induces large shear plastic strain in the region close to the contact surface, which substitutes 
external sliding with an internal sliding within a localized shear band. Shear stress in such a band is equal to the yield strength in shear under the given local pressure.

Variation of the sample thickness at the center with a rising pressure $p$ at the contact surface at the center is plotted in Fig. 8. Initially, when pressure is smaller than $50 \mathrm{GPa}$, the thickness drops very fast with an increasing pressure, after that the rate of thickness reduction slows down. At the pressure $p=285 \mathrm{GPa}$, the thickness is only $2.6 \mu \mathrm{m}$, which is slightly smaller than $3 \mu \mathrm{m}$ in experiments by (Hemley et al., 1997) but larger than $2.2 \mu \mathrm{m}$ in (Merkel et al., 1999). This discrepancy is not critical because of a possible experimental error in determination of such a small thickness at such a high pressure.

Fig. 9 shows the evolving stresses $\sigma_{z z}, \sigma_{z z}-\sigma_{r r}$, and $\tau_{z r}$ with increasing applied stresses. The stress gradients in the radial direction increase with the increase of loads. For normal stresses, the gradient is small along the thickness, almost zero for $\sigma_{z z}$, and slightly larger for $\sigma_{r r}$. Within the plastic sliding zone, which grows with increasing force, $\sigma_{r r}=\sigma_{z z}$ at the contact surface because the friction stress is equal to yield strength in shear, and the contribution of the deviatoric normal stresses is negligible. Therefore, in Fig. 9, $\sigma_{z z}-\sigma_{r r}$ is very small in the region close to the surface, and the difference grows from the contact surface to the symmetry plane. For a small force and in the cohesion or Coulomb slipping zone, $\sigma_{r r} \neq \sigma_{z z}$. The shear stress $\tau_{z r}$ has a much larger gradient along the thickness since it varies from zero at the symmetry plane to the yield strength (in the plastic slipping zone) at the contact surface. Since thickness decreases and the yield strength increases with increasing pressure, both the shear stress $\tau_{z r}$ and its gradient along the thickness direction increase. The radial gradient of $\tau_{z r}$ is quite small.

\subsection{Effect of elastic properties of diamond}

Although there is a good agreement among different studies on the second-order elastic constants, significant discrepancies appear for the third-order elastic constants. For example, the difference in $\mathrm{C}_{456}$ reaches around $3000 \mathrm{GPa}$ in Anastassakis et al. (1990) and Lang and Gupta (2011), and for all of the six third-order constants in Lang and Gupta (2011) and Nielsen (1986)

are more than $1000 \mathrm{GPa}$. While all these third-order elastic constants give a very good 
correspondence with experiments in Lang and Gupta (2011) at pressure below $100 \mathrm{GPa}$ under simple loadings, the differences grows when pressure reaches several megabars or, for example, difference in shear stress is also significant even under $100 \mathrm{GPa}$ under complex loadings. Fig. 4 shows that the pressure distribution is quite consistent with experimental results. However, with the third-order elastic constants from Lang and Gupta (2011), there is no cupping phenomenon (i.e., appearance of a cup-like shape of the contact diamond-sample surface) in Fig. 10a when pressure reaches $285 \mathrm{GPa}$, while it occurred in experiments (Hemley et al., 1997). In Fig. 10b, with the third-order elastic constants from Nielsen (1986), an obvious cupping appears, and the thickness of a sample at the center is $3.2 \mu \mathrm{m}$, which is very close to the thickness of $3.0 \mu \mathrm{m}$ in experiments. In addition, if we use the third-order elastic constants from Nielsen (1986) multiplied by 0.8 , the cupping becomes more obvious and the thickness is a little larger in experiments at the center of a sample. Fig. 5 (b) shows that all three of these cases give very good correspondence with experimental pressure distributions from Hemley et al. (1997). When pressure reaches several megabars, the fourth-order elastic constants in the potential function may also become important. However, there is currently not enough experimental or first principle data to determine all of the 11 fourth-order elastic constants. Fig. 11 plots the pressure distribution and half of a sample thickness variations with an increase in the applied normal stress $\sigma_{n}$. Visible bending of an anvil starts at a pressure of $210 \mathrm{GPa}$ at the center. Cupping starts at a maximum pressure of $260 \mathrm{GPa}$ and becomes very obvious at a maximum pressure of around $300 \mathrm{GPa}$. The thickness at the periphery is still larger than the one at the center, which is consistent with experimental observations in Hemley et al. (1997). With a pressure above $200 \mathrm{GPa}$ at the center, the thickness of the sample at the center only slightly reduces as the pressure continues to rise (Fig. 11b), which was also found in Merkel et al. (2000). The main reason is in the large bending of an anvil and reduction in the sample thicknes and increase in pressure away from the center, which requires a significant increase in force. From a maximum pressure of $210 \mathrm{GPa}$ to $300 \mathrm{GPa}$, the applied normal stress $\sigma_{n}$ nearly doubles. While the entire sample (except for the material at the $z$ axis) plastically flows to the periphery at a lower applied load, above the maximum pressure of $220 \mathrm{GPa}$, the material at the center $(r<10 \mu \mathrm{m})$ is moving towards the $z$ axis, and the deformation in this region is completely elastic during the pressure increase to $300 \mathrm{GPa}$ due to the bending and cupping of diamond. 


\section{Evolution of the stress-strain fields in a diamond anvil}

The deformation of diamond is quite small except for the region contacting the sample. The boundary of the anvil, except with the contact surface of a sample, does not change the shape. Therefore, we will discuss the stress and strain fields close to the contact surface only.

The variation of the distribution of pressure $p$ in the anvil with the growing applied stress $\sigma_{n}$ is shown in Fig. 12. With different third-order elastic constants, the differences in the pressure distribution are not obvious for the region with pressure lower than $100 \mathrm{GPa}$ for different applied stresses. Thus, at $\sigma_{n}=1.652 \mathrm{GPa}$, the pressure distribution is almost the same in the Fig. 12a and b. With a growth of $\sigma_{n}$, the difference increases in the region close to the center of contact surface, where the high pressure region is localized, but for the other regions, the pressure distribution is very similar for both cases. The absolute value of the third-order elastic constants in Lang and Gupta (2011) are larger than those in Nielsen (1986), which causes the increase in material stiffness and stronger stress concentrations. Therefore, the maximum pressure in Fig. 12a is larger than the one in Fig. 12b. Since the diamond stiffening in Fig. 12a also increases the stress concentration in the sample, the pressure in the sample reaches a higher value under the same applied load than for the case in Fig. 12b. Thus, for the same maximum pressure at the center of a sample, the applied $\sigma_{n}$ is larger for the case with a weaker third-order elastic constant in Nielsen (1986) than in Lang and Gupta (2011). The larger $\sigma_{n}$ and less stiff anvil cause the cupping in Fig 10b but not in Fig. 10a.

Similar to the pressure $p$, stress $\sigma_{z z}$ in Fig. 13 decreases from the center of contact surface

to the periphery. In the region with $z>50 \mu \mathrm{m}$, the stress $\sigma_{z z}$ gradient along the $z$ direction is much smaller in comparison with the pressure gradient. Because of diamond stiffening in Fig. 13a, the stress concentration is larger than the one in Fig. 13b.

Fig. 14 shows the evolution of stress $\sigma_{r r}$ with the growth of applied stress. The difference becomes more obvious for larger loads and in the region of the strong stress concentration. In comparison with the $p$ and $\sigma_{z z}$ gradients, the stress $\sigma_{r r}$ gradient along the radial direction in the region with $z>40 \mu m$ is much smaller but the stress $\sigma_{r r}$ gradient along the $z$ direction is larger. The stress $\sigma_{r r}$ concentration is a little more severe in Fig. 14a than Fig. 14b. 
The evolution of shear stress $\tau_{r z}$ in Fig. 15 is governed by the following conditions. Close to the $z$ axis, due to symmetry, the shear stress $\tau_{r z}$ is very small; close to the contact surface, it is also relatively small, because friction stress is limited by the yield strength in shear of the sample. Within an anvil the shear stress reaches a high value of $34 \mathrm{GPa}$, which can cause shear lattice instability, and nucleation of dislocations or cracks. Note that shear strength for diamond, estimated from shock-wave experiments, is in the range 20-35 GPa (Clayton, 2014).

The region with maximum shear stress is significantly shifted from the region of maximum pressure. Since shear stress grows from the contact surface to the interior of a diamond but reduces from the contact surface to the inside of a sample, this can be a reason why, in experiments, the shear stress based on a sample measurement is smaller than that based on diamond measurements (see Fig. 1 in Jeanloz et al., 1991). While for a smaller applied force in Fig. 15 (1) and (2), the effect of the different third-order elastic constants is relatively small and exhibits itself in different sizes of the regions with the maximum shear stress (brown regions in (1) and green regions in (2)). For the largest applied force, Fig. 15 (a) contains the region with the maximum shear stress, which is larger by $2.24 \mathrm{GPa}$ than that in Fig. 15 (b).

Next we discuss the distribution of Eulerian strain $B$ in the anvil and the differences by using the third-order elastic constants. Due to larger third-order elastic constants in Lang and Gupta (2011), there is a larger stress concentration than when using elastic constants from Nielsen (1986). Larger third-order constants cause stiffening and reduce elastic strains for the same stresses, see results for $B_{z z}$ for $\sigma_{n}=1.652 \mathrm{GPa}$ near the contact surface. Remarkably, $B_{z z}$ in diamond reaches 0.15 for $\sigma_{n}=2.546 \mathrm{GPa}$. The strain $B_{z z}$ gradient is large in the radial direction but smaller in the $z$ direction.

The evolution of $B_{r r}$ is presented in Fig. 17. It is interesting that there is a region with tensile strain $B_{r r}$ above the contact surface but the stress $\sigma_{r r}$ is compressive. Since strength of diamond anvils is estimated based on maximum principle tensile strain criterion (Novikov et al., 1994) (which was used in small strain formulation and was not justified for such extreme conditions), fracture of diamond may initiate in this region. In comparison with strain $B_{z z}$, the strain $B_{r r}$ gradient is larger in the $z$ direction but smaller in the radial direction. In Fig. 17a with larger 
stiffening, there is a slightly smaller strain $B_{r r}$ than that in Fig. 17b. The difference in $B_{r r}$ for different third-order constants is less obvious than for $B_{z z}$, because $B_{r r}$ is much smaller than $B_{z z}$.

Fig. 18 shows the distribution of shear strain $B_{r z}$ in the anvil with different third-order constants. Qualitatively, $B_{r z}$ is very close to the distribution of shear stress in Fig. 15, despite the nonlinear elasticity. Shear strain above the contact surface reaches 0.024 . Due to symmetry with respect to the $z$ axis, shear strain is small in the region close to the $z$ axis. Due to limitation of the friction stress by the yield strength in shear of a sample at the contact surface, shear strain is also relatively small close to the contact surface. This causes large gradients in $B_{r z}$. Larger elastic stiffening in Fig. 18a than in Fig.18b due to larger magnitude of the third-order elastic constants causes smaller shear strain.

The effect of the third-order elastic constants of diamond on the stress-strain fields in diamond anvil is summarized in Fig. 19 for the case when the maximum pressure in the sample reaches $285 \mathrm{GPa}$. With elastic softening from (a) to (b) and then to (c), the cupping of the contact surface appears and gets more pronounced, and the applied normal stress grows in order to reach the same maximum pressure in the sample. With approximately the same stresses at the center of the contact surface of the diamond, differences in stresses away from the center increase. Maximum shear stress reaches a huge value of $52 \mathrm{GPa}$, which may cause local plastic relaxation. All strains also increase from (a) to (b) and then to (c), including strains at the center of the contact surface. Maximum shear strains reaches a remarkably large value of 0.04 . These results can be used for optimization of the anvil design for reaching higher pressure in a sample. It is evident that reducing cupping by increasing initial angle of inclination of the contact surface between diamond and sample may reduce the total force for reaching the same pressure and corresponding stresses and strains. This, however, will significantly reduce sample thickness at the center (Merkel et al., 2000), which is undesirable from the experimental point of view.

\section{Concluding remarks}

In this paper, a compression of a sample between two single-crystal diamond anvils in a conventional megabar diamond cell at $300 \mathrm{GPa}$ pressures is modeled, and detailed stress-strain field evolution is obtained for both the sample and diamond. The complexity of the problem arises

from the extreme reduction of a sample thickness by a factor of 30 , accumulated plastic strain up 
to 23, and finite elastic deformation of the sample. Even in the diamond, axial elastic strain reaches 0.17 and the geometry of the contact surface between diamond and a sample changes from convex to concave, exhibiting a cupping phenomenon. Controlling of the shape of the contact surface is crucial for anvil cell designs to reach extreme pressures. Thus, our model includes both large plastic and elastic deformations of the sample, and considers an isotropic polycrystalline material with the nonlinear Murnaghan elasticity law and pressure dependent yield condition. The thirdorder anisotropic elasticity is utilized for the diamond single crystal. The computational algorithm is presented with a detailed description of the radial return algorithms and derivation of the consistent tangent moduli. All material parameters for rhenium, as a typical gasket material, and diamond are calibrated based on the published experimental and first-principle data. Remarkably good correspondence between numerical and experimental pressure distributions at the diamondsample contact surface is obtained for pressure up to $285 \mathrm{GPa}$, the pressures reached in the corresponding experiment, extending the results of previous modeling (Merkel et al., 1999). The shape of the sample and, consequently, diamond-sample contact surface strongly depends on the third-order elastic constants of the diamond. Because of a significant uncertainty in the values of these constants reported in the literature, their effect is studied in detail. Proper third-order elastic constants are found for which the phenomenon of cupping of the diamond-sample contact surface observed in experiments is reproduced. This is crucial for further increase in maximum achievable pressure with single crystal diamond.

The obtained results can be used for development of the coupled experimental and theoretical procedure for extraction of all mechanical fields and properties from the experiments, which may contain strong heterogeneity of all fields. The measured pressure distribution along the contact surface between the sample and diamond, combined with the measured sample thickness under the load, is used to determine the pressure dependence of the yield strength in shear (Jeanloz et al., 1991; Levitas et al. 1996; Sung et al., 1977; Weir et al., 1998). Usually it is done using simplified equilibrium equation (1). Now it can be done much more precisely utilizing iteratively results of the current simulations and fitting not only the pressure distribution but also the shape of the sample to the experiments. Measured components of the elastic strain tensor in some regions of a sample (Duffy et al., 1999b; Hemley et al., 1997; Hemley et al., 2005 Merkel et al., 2013; Nisr et al., 2014; Singh 1993; Singh et al., 2012; Singh et al., 1998; Wenk et al., 2007) and 
diamond can be used in coupling with the current simulations to determine higher order elastic constants.

Further increase in maximum static pressures, achieved in the current experimental setting, continues to be of great interest. Recently, pressures up to $750 \mathrm{GPa}$ have been reported (Dubrovinsky et al., 2015), though the specific pressures achieved remain somewhat controversial because of uncertainties in the pressure calibration as well as in the exact heterogeneous stress state of the samples studied. Our approach can be used for iterative optimal design of diamond anvil cell and loading conditions. There are new developments that involve shaping the anvil tip (e.g., toroid designs (Trojan et al, 2008)) that we do not address and will be considered in a later study. More sophisticated models of plastic flow can be developed and used in the future. Thus, anisotropic elastoplasticity can be used that takes into account texture development and back stresses. Since the thickness of a sample at high pressure is just a few $\mu \mathrm{m}$ and radial gradients of all fields are very large, a gradient-type theory of plasticity should be developed for these purposes. It can be based on phenomenological strain gradient theories (Clayton, 2011; Fleck and Hutchinson, 1993; Lubarda, 2016; Martinez-Paneda and Niordson, 2015; Polizzotto, 2009), dislocation mechanism-based theories (Gao et al., 1999; Huang et al., 2000; Hwang et al., 2003),

or phase field theory for discrete dislocations (Levitas and Javanbakht, 2015b; Javanbakht and Levitas, 2016). The next step will be allowing for interaction between plasticity and phase transformation with the goal to study phase transformation and seek new phases with unprecedented properties, using the development of approaches in Feng and Levitas (2013), Feng et al. (2014), Levitas and Zarechnyy (2010a, 2010b), or phase field approaches in Javanbakht and Levitas( 2015) and Levitas and Javanbakht (2014; 2015a).

\section{Acknowledgements}

VIL and BF gratefully acknowledge the support of NSF (DMR-1434613), ARO (W911NF12-1-0340), DARPA (W31P4Q-13-1-0010), WDC Research Fund of the Carnegie Institution of Washington, and Iowa State University (Schafer 2050 Challenge Professorship). This work was initiated when VIL was on sabbatical leave at the Geophysical Laboratory of Carnegie Institution of Washington. VIL appreciates the hospitality of R. J. Hemley and many other staff members. RJH acknowledges support from the DOE/NNSA (CDAC, DE-NA-0002006), the DOE Office of Science (EFree, DE-SC-0001057), the Rose Foundation, and the WDC Research Fund. This work is a contribution to the Deep Carbon Observatory. 


\section{References}

1. ABAQUS V6.11, Abaqus User Subroutines Reference Manual: UMAT. Providence PI, USA: Abaqus INC (2011)

2. Adams, D. M., and Shaw, A. C., 1982. A computer-aided-design study of the behavior of diamond anvils under stress. J. of Phys. D-Appl. Phys. 15, 1609-1635.

3. Anastassakis, E., Cantarero, A., and Cardona, M., 1990. Piezo-Raman Measurements and Anharmonic Parameters in Silicon and Diamond. Phys. Rev. B 41, 7529-7535.

4. Bridgman, P. W., 1952. Studies in large plastic flow and fracture with special emphasis on the effects of hydrostatic pressure. McGraw-Hill, New York.

5. Bruno, M. S., and Dunn, K. J., 1984. Stress-analysis of a beveled diamond anvil. Rev. Sci. Instrum. 55, 940-943.

6. Clayton, J. D. 2011. Nonlinear Mechanics of Crystals. Springer Publishers, New York.

7. Clayton, J. D. 2013. Nonlinear Eulerian thermoelasticity for anisotropic crystals. J. Mech. Phys. Solids 61, 19832014.

8. Clayton, J. D. 2014. Analysis of shock compression of strong single crystals with logarithmic thermoelasticplastic theory. Int. J. Eng. Sci. 79, 1-20.

9. Cousins, C. S. G., 2003. Elasticity of carbon allotropes. I. Optimization, and subsequent modification, of an anharmonic Keating model for cubic diamond. Phys. Rev. B 67, 024107.

10. Dubrovinsky, L., Dubrovinskaia, N., Prakapenka, V. B., and Abakumov, A. M., 2012. Implementation of microball nanodiamond anvils for high-pressure studies above 6 Mbar. Nat. Commun. 3, 1163.

11. Dubrovinsky, L. Dubrovinskaia, N, Bykova, E, et al. 2015. The most incompressible metal osmium at static pressures above 750 gigapascals. Nature 525, 226-229.

12. Duffy, T. S., Shen, G. Y., Heinz, D. L., Shu, J. F., Ma, Y. Z., Mao, H. K., Hemley, R. J., and Singh, A. K., 1999a. Lattice strains in gold and rhenium under nonhydrostatic compression to 37 GPa. Phys. Rev. B 60, 15063-15073.

13. Duffy, T. S., Shen, G. Y., Shu, J. F., Mao, H. K., Hemley, R. J., and Singh, A. K., 1999b. Elasticity, shear strength, and equation of state of molybdenum and gold from x-ray diffraction under nonhydrostatic compression to 24 GPa. J. Appl. Phys. 86, 6729-6736.

14. Dunne, F., Petrinic, N., 2005. Introduction to computational plasticity. Oxford University, Oxford; New York.

15. Eremets, M. I., 1996. High pressure experimental methods. Oxford University, Oxford; New York.

16. Feng, B., and Levitas, V. I., 2013. Coupled phase transformations and plastic flows under torsion at high pressure in rotational diamond anvil cell: Effect of contact sliding. J. Appl. Phys. 114, 213514.

17. Feng, B., Levitas, V. I., and Ma, Y. Z., 2014. Strain-induced phase transformation under compression in a diamond anvil cell: Simulations of a sample and gasket. J. Appl. Phys. 115, 163509.

18. Feng, B., Levitas, V. I., and Zarechnyy, O. M., 2013. Plastic flows and phase transformations in materials under compression in diamond anvil cell: effect of contact sliding. J. Appl. Phys. 114, 043506.

19. Fish, J., and Shek, K., 1999. Computational aspects of incrementally objective algorithms for large deformation plasticity. Int. J. Numer. Methods Eng. 44, 839-851.

20. Fleck, N. A., and Hutchinson, J. W., 1993. A Phenomenological Theory for Strain Gradient Effects in Plasticity. J. Mech. Phys. Solids 41, 1825-1857.

21. Gao, H., Huang, Y., Nix, W. D., and Hutchinson, J. W., 1999. Mechanism-based strain gradient plasticity - I. Theory. J. Mech. Phys. Solids 47, 1239-1263.

22. Goettel, K. A., Mao, H., and Bell, P. M., 1985. Generation of static pressures above 2.5 megabars in a diamondanvil pressure cell. Rev. Sci. Instrum. 56, 1420-1427.

23. Grimsditch, M. H., Anastassakis, E., and Cardona, M., 1978. Effect of uniaxial stress on zone-center optical phonon of diamond. Phys. Rev. B 18, 901-904.

24. Hemley, R. J., Mao, H. K., Shen, G. Y., Badro, J., Gillet, P., Hanfland, M., and Hausermann, D., 1997. X-ray imaging of stress and strain of diamond, iron, and tungsten at megabar pressures. Science 276, 1242-1245. 
25. Hemley, R. J., Mao, H. K., and Struzhkin, V. V., 2005. Synchrotron radiation and high pressure: new light on materials under extreme conditions. J. Synchrot. Radiat. 12, 135-154.

26. Hill, R., 1950. The mathematical theory of plasticity. Clarendon Press, Oxford.

27. Huang, Y., Gao, H., Nix, W. D., and Hutchinson, J. W., 2000. Mechanism-based strain gradient plasticity - II. Analysis. J. Mech. Phys. Solids 48, 99-128.

28. Hwang, K. C., Jiang, H., Huang, Y., and Gao, H., 2003. Finite deformation analysis of mechanism-based strain gradient plasticity: torsion and crack tip field. Int. J. Plast. 19, 235-251.

29. Idesman, A. V., and Levitas, V. I., 1995. Finite-Element Procedure for Solving Contact Thermoelastoplastic Problems at Large Strains, Normal and High-Pressures. Comput. Meth. Appl. Mech. Eng. 126, 39-66.

30. Javanbakht, M., and Levitas, V. I., 2015. Ineraction between phase transformations and dislocations at the nanoscale. Part 2. Phase field simulation examples. J. Mech. Phys. Solids 82, 164-185.

31. Javanbakht, M., and Levitas, V. I., 2016. Phase field approach to dislocation evolution at large strains: computational aspects. Int. J. Solids and Struct. 2016, 82, 95-110.

32. Jeanloz, R., Godwal, B. K., and Meade, C., 1991. Static strength and equation of state of rhenium at ultra-high pressures. Nature 349, 687-689.

33. Khoei, A. R., and Bakhshiani, A., 2004. A hypoelasto-plastic finite strain simulation of powder compaction processes with density-dependent endochronic model. Int. J. Solids Struct. 41, 6081-6110.

34. Lang, J. M., and Gupta, Y. M., 2011. Experimental Determination of Third-Order Elastic Constants of Diamond. Phys. Rev. Lett. 106.

35. Lubarda, V. A., 2016. On the recoverable and dissipative parts of higher order stresses in strain gradient plasticity. Int. J. Plast. 78, 26-43.

36. Levitas, V. I., 1981. Stressed state of a reaction container of a recessed anvil-type hig pressure chamber. J. Superhard Mater. 3, 9-13.

37. Levitas, V. I., 1996. Large Deformation of materials with complex rheological properties at normal and high pressure. Nova Science Publishers, New York.

38. Levitas, V. I., and Javanbakht, M., 2015a. Ineraction between phase transformations and dislocations at the nanoscale. Part 1. General phase field approach. J. Mech. Phys. Solids, 82, 287-319.

39. Levitas, V. I., and Javanbakht, M., 2014. Phase transformations in nanograin materials under high pressure and plastic shear: nanoscale mechanism. Nanoscale 6, 162-166.

40. Levitas, V. I., and Javanbakht, M., 2015b. Thermodynamically consistent phase field approach to dislocation evolution at small and large strains. J. Mech. Phys. Solids, 82, 345-366.

41. Levitas, V. I., Ma, Y. Z., Hashemi, J., Holtz, M., and Guven, N., 2006. Strain-induced disorder, phase transformations, and transformation-induced plasticity in hexagonal boron nitride under compression and shear in a rotational diamond anvil cell: In situ X-ray diffraction study and modeling. J. Chem. Phys. 125, 044507.

42. Levitas, V. I., Polotnyak, S. B., and Idesman, A. V., 1996. Large elastoplastic strains and stress state of deformable gasket of high pressure equipment with diamond anvils. Strength Mater 3, 221-227.

43. Levitas, V. I., Shestakov, S. I., and Borimskiy, A. I., 1986. Investigation of stressed-strained state of cemented carbide and steel matrices of high pressure apparatus of cylindrical type. High Press. Phys. Technol. 7, 70-73.

44. Levitas, V. I., Stashkevich I.E., and Nemirovskiy A. B., 1994. Stress-strain diagram of metals under large uniform compressive strains. Strength Mater26, 676-680.

45. Levitas, V. I., and Zarechnyy, O. M., 2010a. Modeling and simulation of strain-induced phase transformations under compression and torsion in a rotational diamond anvil cell. Phys. Rev. B 82, 174124.

46. Levitas, V. I., and Zarechnyy, O. M., 2010b. Modeling and simulation of strain-induced phase transformations under compression in a diamond anvil cell. Phys. Rev. B 82, 174123.

47. Levitas, V. I., and Zarechnyy, O. M., 2010c. Numerical study of stress and plastic strain evolution under compression and shear of a sample in a rotational anvil cell. High Pressure Res. 30, 653-669.

48. Li, L., Weidner, D. J., Chen, J. H., Vaughan, M. T., Davis, M., and Durham, W. B., 2004. X-ray strain analysis at high pressure: Effect of plastic deformation in MgO. J. Appl. Phys. 95, 8357-8365. 
49. Lubarda, V. A., 2001. Elastoplasticity theory. CRC Press LLC, Boca Raton, Florida.

50. Lurie, A. I., 1990. Nonlinear theory of elasticity. Elsevier Science Pub. Co., New York.

51. Lv, M. B., Cheng, Y., Qi, Y. Y., Ji, G. F., and Piao, C. G., 2012. Elastic properties and phonon dispersions of rhenium in hexagonal-close-packed structure under pressure from first principles. Physica B 407, 778-783.

52. Manghnani, M. H., Katahara, K., and Fisher, E. S., 1974. Ultrasonic equation of state of rhenium. Phys. Rev. B 9, 1421-1431.

53. Mao, H. K., and Bell, P. M., 1978. High-pressure physics-sustained static generation of 1.36 to 1.72 megabars. Science 200, 1145-1147.

54. Martinez-Paneda, E., and Niordson, C. F. 2015. On fracture in finite strain gradient plasticity. Int. J. Plast. 80, 154-167.

55. McSkimin, H. J., and Andreatc. P, 1972. Elastic-moduli of diamond as a function of pressure and temperature. J. Appl. Phys. 43, 2944-2948.

56. Meade, C., and Jeanloz, R., 1988. Effect of a coordination change on the strength of amorphous sio2. Science 241, 1072-1074.

57. Merkel, S., Hemley, R. J., and Mao, H. K., 1999. Finite-element modeling of diamond deformation at multimegabar pressures. Appl. Phys. Lett. 74, 656-658.

58. Merkel, S., Hemley, R. J., and Mao, H. K., Teter, D.M., 2000. Finite-element modeling and ab initio calculations of megabar stresses in the diamond anvil cell. Proceedings of the conference AIRAPT-XVII, pp. 68-73.

59. Merkel, S., Liermann, H. P., Miyagi, L., and Wenk, H. R., 2013. In situ radial x-ray diffraction study of texture and stress during phase transformations in bcc-, fcc- and hcp-iron up to $36 \mathrm{GPa}$ and $1000 \mathrm{~K}$. Acta Mater. 61, 5144-5151.

60. Moss, W. C., and Goettel, K. A., 1987. Finite-element design of diamond anvils. Appl. Phys. Lett. 50, 25-27.

61. Moss, W. C., Hallquist, J. O., Reichlin, R., Goettel, K. A., and Martin, S., 1986. Finite-element analysis of the diamond anvil cell - achieving 4.6 Mbar. Appl. Phys. Lett. 48, 1258-1260.

62. Murnaghan, F. D. 1951. Finite deformation of an elastic solid. John Wiley \& Sons, INC. New York.

63. Nielsen, O. H., 1986. Optical phonons and elasticity of diamond at megabar stresses. Phys. Rev. B 34, 5808-5819.

64. Nisr, C., Ribarik, G., Ungar, T., Vaughan, G. B. M., and Merkel, S., 2014. Three-dimensional X-ray diffraction in the diamond anvil cell: application to stishovite. High Pressure Res. 34, 158-166.

65. Novikov, N. V., 2005. Synthesis of superhard materials. J. Mater. Proces. Technol. 161, 169-172.

66. Novikov, N. V., and Levitas, V. I., 1985. Simulation of thermoplastic flow of materials in high pressure apparatus. Bulletin of the Ukrainian SSR Academy of Sciences 8, 7-17.

67. Novikov, N. V., Levitas, V. I., and Idesman, A. V., 1990. Theoretical description of thermomechanical effects in high pressure apparatus. High Pressure Res. 5, 868-870.

68. Novikov, N. V., Levitas, V. I., Leshchuk, A. A., and Idesman, A. V., 1991a. Mathematical modeling of diamond synthesis process. High Pressure Res. 7, 195-197.

69. Novikov, N. V., Levitas, V. I., and Polotnyak, S. B., 1987. Numerical simulation of the stressed-strained and limiting states of the elements of high pressure apparatus with diamond anvils. J. Superhard Mater. 9, 1-9.

70. Novikov, N. V., Levitas, V. I., Polotnyak, S. B., and Potyomkin, M. M., 1992. Numerical optimization of diamond anvil cell design. High Pressure Res. 8, 507-509.

71. Novikov, N. V., Levitas, V. I., Polotnyak, S. B., and Potyomkin, M. M., 1994. Numerical method for optimizing the design of a high-pressure apparatus with diamond anvils. Strength Mater-Engl. Tr. 26, 294-302.

72. Novikov, N. V., Levitas, V. I., and Shestakov, S. I., 1986. Fundamentals of Strength and Durability Calculations for High-Pressure Apparatus Elements. Physica B+C 139, 782-784.

73. Novikov, N. V., Levitas, V. I., and Shestakov, S. I., 1991b. Numerical modeling of strength and longevity of structures with allowance for scale effect. Report 1 . Substrantiation of strength and longevity criteria. Strength Mater-Engl. Tr. 5, 527-533. 
74. Novikov, N. V., Levitas, V. I., and Shestakov, S. I., 1991c. Numerical modeling of strength and longevity of structures with allowance for scale effect. Report 2. Investigation of the strength and longevity of hard-alloy die for high pressure apparatus. Strength Mater-Engl. Tr. 6, 635-642.

75. Novikov, N. V., Levitas, V. I., and Shestakov, S. I., 1991d. Numerical modeling of strength and longevity of structures with allowance for scale effect. Report 3. Investigation of the stressed state, strength and longevity of cylindrical-type high-pressure apparatus. Strength Mater-Engl. Tr. 6, 644-651.

76. Novikov, N. V., Polotnyak S.B., Shvedov L.K., and Levitas, V.I., 1999, Phase transitions under compression and shear in diamond anvils: Experiment and theory. J. Superhard Mater. 3, 39-51.

77. Polizzotto, C., 2009. A nonlocal strain gradient plasticity theory for finite deformations. Int. J. Plast. 25, 12801300.

78. Ponthot, J. P., 2002. Unified stress update algorithms for the numerical simulation of large deformation elastoplastic and elasto-viscoplastic processes. Int. J. Plast. 18, 91-126.

79. Simo, J., Hughes, T. J. R., 1998. Computational inelasticity. Springer, New York.

80. Singh, A. K., 1993. The lattice strains in a specimen (cubic system) compressed nonhydrostatically in an opposed anvil device. J. Appl. Phys. 73, 4278-4286.

81. Singh, A. K., Hu, J., Shu, J., Mao, H. K., and Hemley, R. J., 2012. Strength of rhenium from x-ray diffraction experiments under nonhydrostatic compression to $250 \mathrm{GPa}$. J. Phys. Conf. Series, 377, 012008.

82. Singh, A. K., Mao, H. K., Shu, J. F., and Hemley, R. J., 1998. Estimation of single-crystal elastic moduli from polycrystalline X-ray diffraction at high pressure: Application to FeO and iron. Phys. Rev. Lett. 80, 2157-2160.

83. Sung, C. M., Goetze, C., and Mao, H. K., 1977. Pressure Distribution in diamond anvil press and shear-strength of fayalite. Rev. Sci. Instrum. 48, 1386-1391.

84. Thomsen, E. G., Yang, C. T., and Kobayashi, S. O., 1965. Mechanics of plastic deformation in metal processing. Macmillan, New York.

85. Trojan I. A., Eremets, M. I., Medvedev, S. A., Gavriliuk, A. G., and Prakapenka, V. B., 2008. Transformation from molecular to polymeric nitrogen at high pressures and temperatures: In stitu X-ray diffraction study. Appl. Phys. Lett. 93, 091907.

86. Vohra, Y. K., Duclos, S. J., Brister, K. E., and Ruoff, A. L., 1988. Static pressure of 255-GPa (2.55-Mbar) by xray-diffraction - comparison with extrapolation of the ruby pressure scale. Phys. Rev. Lett. 61, 574-577.

87. Vohra, Y. K., Duclos, S. J., and Ruoff, A. L., 1987. High-pressure x-ray-diffraction studies on rhenium up to 216 GPa (2.16 Mbar). Phys. Rev. B 36, 9790-9792.

88. Voronin, G. A., Mal'nev, V. I., and Nevstruev, G., F., 1984. Effect of inclusions on the strength of synthetic diamonds at high pressure. J. Superhard Mater. 2, 33-37.

89. Voyiadjis, G. Z., and Abed, F. H., 2006. Implicit algorithm for finite deformation hypoelastic-viscoplastici in fcc metals. Int. J. Numer. Meth. Eng. 67, 933-959.

90. Weir, S. T., Akella, J., Ruddle, C., Goodwin, T., and Hsiung, L., 1998. Static strengths of Ta and U under ultrahigh pressures. Phys. Rev. B 58, 11258-11265.

91. Wenk, H. R., Huensche, I., and Kestens, L., 2007. In-situ observation of texture changes during phase transformations in ultra-low-carbon steel. Metall. Mater. Trans. 38A, 261-267.

92. Zhao, Y. and Zhang. J., 2007. Enhancement of yield strength in zirconium metal through high-pressure induced structural phase transition. In-situ observation of texture changes during phase transformations in ultra-lowcarbon steel. Metall. Mater. Appl. Phys. Lett. 91, 201907. 
Appendix. Explicit expressions for $\frac{\partial \boldsymbol{f}\left(\boldsymbol{B}_{e}\right)}{\partial \boldsymbol{B}_{e}}, \frac{\partial \varphi}{\partial \boldsymbol{\sigma}}$ and $\frac{\partial \varphi}{\partial q}$.

1. With the help of the elasticity law (29), $\frac{\partial \boldsymbol{f}\left(\boldsymbol{B}_{e}\right)}{\partial \boldsymbol{B}_{e}}$ can be evaluated as follows:

$$
\begin{aligned}
& \frac{\partial \sigma_{i j}}{\partial B_{e_{l j}}}=\frac{\partial(1 / \operatorname{det} \boldsymbol{F})}{\partial B_{e_{l f}}} \sigma_{i j} \operatorname{det} \boldsymbol{F}+\frac{2 \delta_{i k}}{\operatorname{det} \boldsymbol{F}}\left(\lambda I_{1} \delta_{f j}+2 \mu B_{e_{f j}}+\left(l I_{1}^{2}-2 m I_{2}\right) \delta_{f j}+n \frac{\partial I_{3}}{\partial B_{e_{j j}}}+2 m I_{1} B_{e_{f j}}\right)+ \\
& (1 / \operatorname{det} \boldsymbol{F})\left(2 B_{e_{i j}}+\delta_{i j}\right)\left[\lambda \delta_{k f}+2 l \delta_{k f} I_{1}-2 m\left(I_{1} \delta_{k f}-B_{e_{k f}}\right)\right]+ \\
& (1 / \operatorname{det} \boldsymbol{F})\left(2 B_{e_{i k}}+\delta_{i k}\right)\left(2 \mu \delta_{j f}+2 m \delta_{j f} I_{1}\right)+(1 / \operatorname{det} \boldsymbol{F})\left(2 B_{e_{i m}}+\delta_{i m}\right)\left(n \frac{\partial^{2} I_{3}}{\partial B_{e_{n j}} \partial B_{e_{l f}}}+2 m \delta_{j f} B_{e_{n j}}\right) .
\end{aligned}
$$

Tensor $\frac{\partial I_{3}}{\partial \boldsymbol{B}_{e}}$ is given in Eq. (29), and it is easy to obtain $\frac{\partial^{2} I_{3}}{\partial B_{e_{n j}} \partial B_{e_{k f}}}$ by further differentiation.

Tensor $\frac{\partial(1 / \operatorname{det} \boldsymbol{F})}{\partial B_{e_{\text {kf }}}}$ can be obtained from the following procedures. First,

$$
\begin{gathered}
(1 / \operatorname{det} \boldsymbol{F})=\left(\operatorname{det} \boldsymbol{F}^{t} \operatorname{det} \boldsymbol{F}\right)^{-0.5}=\left[\operatorname{det}\left(\boldsymbol{F}^{t} \cdot \boldsymbol{F}\right)\right]^{-0.5}=\left[\operatorname{det}\left(\boldsymbol{I}+2 \boldsymbol{B}_{e}\right)\right]^{-0.5} \\
=\left[\left(1+2 B_{e 1}\right)\left(1+2 B_{e 2}\right)\left(1+2 B_{e 3}\right)\right]^{-0.5}=\left(1+2 I_{1}+4 I_{2}+8 I_{3}\right)^{-0.5},
\end{gathered}
$$

in which $B_{e 1}, B_{e 2}$, and $B_{e 3}$ are the three principle values of tensor $\boldsymbol{B}_{e}$, and $I_{i}$ are the three invariants of the strain tensor $\boldsymbol{B}_{e}$, see Eq.(28). Then we calculate

$$
\frac{\partial(1 / \operatorname{det} \boldsymbol{F})}{\partial \boldsymbol{B}_{e}}=\frac{\partial\left(1+2 I_{1}+4 I_{2}+8 I_{3}\right)^{-0.5}}{\partial \boldsymbol{B}_{e}}=\frac{-1}{\operatorname{det} \boldsymbol{F}^{3}}\left(\boldsymbol{I}+2\left(I_{1} \boldsymbol{I}-\boldsymbol{B}_{e}\right)+4 \frac{\partial I_{3}}{\partial \boldsymbol{B}_{e}}\right),
$$

which concludes evaluation of $\frac{\partial \boldsymbol{f}\left(\boldsymbol{B}_{e}\right)}{\partial \boldsymbol{B}_{e}}$.

2. For linear pressure dependence of the yield surface, Eq.(23), we obtain

$$
\frac{\partial \varphi}{\partial \boldsymbol{\sigma}}=\frac{\sqrt{1.5} \boldsymbol{s}}{\sqrt{\boldsymbol{s}: \boldsymbol{s}}}-\frac{b}{3} \boldsymbol{I} \Phi(q) ; \quad \frac{\partial \varphi}{\partial q}=-(1+b p) \Phi^{\prime}(q),
$$

where $\Phi^{\prime}(q)=\frac{2}{m}\left(\sigma_{y}^{u}-\sigma_{y 0}\right)\left(1-\frac{q}{m}\right)$ for $\frac{q}{m} \leq 1 ; \quad \Phi^{\prime}(q)=0$ for $q>m$. 


\section{Figure Captions.}

Fig. 1. Shear strength in experiments and approximations in our current simulations and in Jeanloz et al. (1991). Solid symbols without error bar are experimental data calibrated from sample pressure gradient, and open symbols with error bar are experimental data calibrated from diamond anvil measurements. The solid and dashed lines are the approximations of experimental data in our current simulations and in Jeanloz et al. (1991).

Fig. 2. (a) Diamond anvil cell scheme, (b) a quarter of the sample and anvil in the initial undeformed state and the geometric parameters of an anvil, and (c) the geometric parameters of a sample.

Fig. 3 Mesh-independence of FEM solutions. (a) Fine mesh with 3198 elements in a sample and (b) rough mesh with 919 elements. Pressure distribution in the sample for fine mesh (c) and rough mesh (d). (e) Pressure distributions at the contact surface for fine and rough meshes.

Fig. 4 Pressure distribution in the sample $(0 \leq r \leq 164 \mu \mathrm{m})$ with an increasing applied normal stress $\sigma_{n}$. The normal stress $\sigma_{n}$ is $0(1), 1.022 \mathrm{GPa}(2), 1.652 \mathrm{GPa}(3), 1.903 \mathrm{GPa}(4)$, and 2.546 $\mathrm{GPa}(5)$.

Fig. 5. Pressure distributions at the contact surface. Lines with symbols are the current simulation results with different third-order elastic constants of diamond, which are taken from Lang and Gupta (2011) in (a) and from Lang and Gupta (2011), Nielsen (1986) and 0.8 of the third-order constants from (Nielsen, 1986) in (b)); black solid lines are experimental data from Hemley et al. (1997); the green dashed line is the simulation results from Merkel et al. (1999).

Fig. 6. Distributions of the shear friction stress $\tau$ in (a), yield strength in shear $\tau_{y}$ and $\mu \sigma_{c}$ at the contact surface at different applied normal stresses $\sigma_{n} . \sigma_{c}$ is the normal contact stress at the contact surface between the diamond and the sample. The shear friction stress in the sliding region $\tau=\min \left(\mu \sigma_{c}, \tau_{y}(p)\right)$, i.e., there is the Coulomb sliding at the periphery and the plastic sliding at the center. In the small cohesion zone (shown for the smallest $\sigma_{n}$ only) $\tau<\min \left(\mu \sigma_{c}, \tau_{y}(p)\right)$. 
Fig. 7. Distribution of the accumulated plastic strain $q$ in the sample $(0 \leq r \leq 164 \mu \mathrm{m})$ with an increasing applied normal stress $\sigma_{n}: 1.022 \mathrm{GPa}(1), 1.652 \mathrm{GPa}(2), 1.903 \mathrm{GPa}(3)$, and 2.546 $\mathrm{GPa}$ (4). The zoomed central part of a sample is shown above the sample for each applied normal stress.

Fig. 8. The variation of the sample thickness $h$ at the center with growing pressure $p$ at the contact surface at the center. The third-order elastic constants are taken from Lang and Gupta (2011). The curve in (b) is a part of the curve in (a) for the pressure $p>60 \mathrm{GPa}$.

Fig. 9 Distributions of stresses $\sigma_{z z}, \sigma_{z z}-\sigma_{r r}$, and $\tau_{z r}$ in the sample $(0 \leq r \leq 164 \mu \mathrm{m})$ with an increasing applied stresses. The applied normal stress $\sigma_{n}$ at the top surface of the diamond in Fig. 2 (a) is $1.022 \mathrm{GPa}(1), 1.652 \mathrm{GPa}(2), 1.903 \mathrm{GPa}(3)$, and $2.546 \mathrm{GPa}$ (4).

Fig. 10. Pressure distribution in the sample $(0 \leq r \leq 164 \mu \mathrm{m})$ when the pressure in the center reaches $285 \mathrm{GPa}$. The third-order elastic constants are taken from Lang and Gupta (2011) in (a), from Nielsen (1986) in (b), and 0.8 of the third-order constants from (Nielsen, 1986) in (c). The thickness of a sample at the center for (a), (b), and (c) is 2.6, 3.2, and 3.6 $\mu \mathrm{m}$.

Fig. 11. Pressure distributions (a) at the contact surface of sample with an increasing applied normal stress $\sigma_{n}$ and half of a thickness of the sample (b) corresponding pressure curves in (a) designated with the same symbols and colors. The third-order elastic constants are taken as 0.8 of the third-order constants from Nielsen (1986).

Fig. 12 Pressure distribution in the anvil with the growth of applied loading obtained with thirdorder elastic constants from Lang and Gupta (2011) (a) and Nielsen (1986) (b). The applied normal stress $\sigma_{n}$ is $1.652 \mathrm{GPa}(1), 1.903 \mathrm{GPa}(2)$, and $2.546 \mathrm{GPa}$ (3). Points $\mathrm{C}$ and $\mathrm{G}$ in the undeformed state (the green figure) corresponds to the Points $\mathrm{C}$ and G in Fig. 2 (b).

Fig. 13 Distribution of stress $\sigma_{z z}$ in the anvil with the growth of applied loading obtained with third-order elastic constants from Lang and Gupta (2011) (a) and Nielsen (1986) (b). The applied normal stress $\sigma_{n}$ is $1.652 \mathrm{GPa}(1), 1.903 \mathrm{GPa}(2)$, and $2.546 \mathrm{GPa}(3)$.

Fig. 14 Distribution of stress $\sigma_{r r}$ in the anvil with the growth of applied loading obtained with third-order elastic constants from Lang and Gupta (2011) (a) and Nielsen (1986) (b). The applied normal stress $\sigma_{n}$ is $1.652 \mathrm{GPa}(1), 1.903 \mathrm{GPa}(2)$, and $2.546 \mathrm{GPa}(3)$. 
Fig. 15. Distribution of shear stress $\tau_{r z}$ in the anvil with the growth of applied loading obtained with the third-order elastic constants from Lang and Gupta (2011) (a) and Nielsen (1986) (b). The applied normal stress $\sigma_{n}$ is $1.652 \mathrm{GPa}(1), 1.903 \mathrm{GPa}(2)$, and $2.546 \mathrm{GPa}$ (3).

Fig. 16. Distribution of strain $B_{z z}$ in the anvil with the growth of applied loading obtained with the third-order elastic constants from Lang and Gupta (2011) (a) and Nielsen (1986) (b). The applied normal stress $\sigma_{n}$ is $1.652 \mathrm{GPa}(1), 1.903 \mathrm{GPa}(2)$, and $2.546 \mathrm{GPa}(3)$.

Fig. 17 Distribution of strain $B_{r r}$ in the anvil with the growth of applied loading obtained with the third-order elastic constants from Lang and Gupta (2011) (a) and Nielsen (1986) (b). The applied normal stress $\sigma_{n}$ is $1.652 \mathrm{GPa}(1), 1.903 \mathrm{GPa}(2)$, and $2.546 \mathrm{GPa}(3)$.

Fig. 18 Distribution of shear strain $B_{r z}$ in the anvil with the growth of applied loading obtained with the third-order elastic constants from Lang and Gupta (2011) (a) and Nielsen (1986) (b). The applied normal stress $\sigma_{n}$ is $1.652 \mathrm{GPa}(1), 1.903 \mathrm{GPa}(2)$, and $2.546 \mathrm{GPa}$ (3).

Fig. 19 Distributions of pressure $p$, stresses, $\sigma_{z z}, \sigma_{r r}$ and $\tau_{z r}$, and strains $B_{z z}, B_{r r}$, and $B_{r z}$ in the anvil when the maximum pressure in the sample reaches $285 \mathrm{GPa}$. Results are obtained with the third-order elastic constants from Lang and Gupta (2011) (a) and Nielsen (1986) (b), and 0.8 of the third-order elastic constants of from Nielsen (1986) (c). The resultant force $F$ is $8818 \mathrm{~N} \mathrm{(a)}, 11061 \mathrm{~N}(\mathrm{~b})$, and $13221 \mathrm{~N}$ (c). The applied normal stress $\sigma_{n}$ is $2.546 \mathrm{GPa}$ (a), $3.124 \mathrm{GPa}(2)$, and $3.817 \mathrm{GPa}(\mathrm{c})$. 


\section{Flowchart for the radial return algorithm}

1. Known: $\overline{\boldsymbol{F}}_{p(n)}, \boldsymbol{\sigma}_{n}, q_{n}, \Delta t$ and $F_{n+1}$ at $n+1$ time step Find: $\boldsymbol{\sigma}_{n+1}, q_{n+1}$, and $\overline{\boldsymbol{F}}_{p(n+1)}$.

$$
\begin{aligned}
& \text { 2. Compute } \boldsymbol{s}_{n+1}^{0}, p_{n+1}^{0} \text {, and } q_{n+1} \text { using following equations } \\
& \left(\begin{array}{l}
F_{e}^{0}=F_{n+1} \cdot \overline{\boldsymbol{F}}_{p n}^{-1}=V_{e(n+1)}^{0} \cdot R_{e(n+1)}^{0} ; \quad \overline{\boldsymbol{F}}_{p(n+1)}^{0}=V_{e(n+1)}^{0} \cdot \boldsymbol{F}_{n+1} ; \\
\boldsymbol{B}_{e(n+1)}^{0}=\frac{1}{2}\left(\boldsymbol{F}_{e}^{0} \cdot \boldsymbol{F}_{e}^{t 0}-\boldsymbol{I}\right) ; \quad \boldsymbol{\sigma}_{n+1}^{0}=\frac{\left(2 \boldsymbol{B}_{e(\mathrm{n}+1)}^{0}+\boldsymbol{I}\right)}{\operatorname{det} \boldsymbol{F}_{n+1}} \cdot \frac{\partial \Psi\left(\boldsymbol{B}_{e(\mathrm{n}+1)}^{0}\right)}{\partial \boldsymbol{B}_{e(\mathrm{n}+1)}^{0}} ; \\
p_{n+1}^{0}=-\frac{1}{3} \boldsymbol{\sigma}_{n+1}^{0}: \boldsymbol{I} ; \quad \boldsymbol{s}_{n+1}^{0}=\boldsymbol{\sigma}_{n+1}^{0}+p_{n+1}^{0} \boldsymbol{I} ; \quad q_{n+1}=q_{n}
\end{array}\right)
\end{aligned}
$$

$$
\begin{aligned}
& \text { If } \varphi\left(\boldsymbol{s}_{n+1}^{0}, p_{n+1}^{0}, q_{n}\right)<0 \\
& \text { save } \boldsymbol{\sigma}_{n+1}, q_{n+1}, \text { and } \\
& \overline{\boldsymbol{F}}_{p(n+1)}, \text { and EXIT. }
\end{aligned}
$$

\section{Evaluate yield condition If $\varphi\left(s_{n+1}^{0}, p_{n+1}^{0}, q_{n}\right) \geq 0$}

4. Update $q_{n+1}$ and $\sigma_{n+1}$, and solve for $\boldsymbol{V}_{e(\mathrm{n}+1)}$ by equations: $\left(\boldsymbol{B}_{e(\mathrm{n}+1)}^{(\mathrm{i}+1)}=\boldsymbol{B}_{e(\mathrm{n}+1)}^{(\mathrm{i})}-\lambda \boldsymbol{V}_{e(\mathrm{n}+1)}^{(\mathrm{i})} \cdot \frac{s_{\mathrm{n}+1}^{(\mathrm{i})}}{\sqrt{s_{\mathrm{n}+1}^{(\mathrm{i})}: s_{\mathrm{n}+1}^{(\mathrm{i})}}} \cdot \boldsymbol{V}_{e(\mathrm{n}+1)}^{(\mathrm{i})} \Delta t ;\right.$ $\boldsymbol{\sigma}_{n+1}^{(\mathrm{i}+1)}=\frac{1}{\operatorname{det} \boldsymbol{F}_{n+1}}\left(2 \boldsymbol{B}_{e(\mathrm{n}+1)}^{(\mathrm{i}+1)}+\boldsymbol{I}\right) \frac{\partial \Psi\left(\boldsymbol{B}_{e(\mathrm{n}+1)}^{(\mathrm{i}+1)}\right)}{\partial \boldsymbol{B}_{e(\mathrm{n}+1)}^{(\mathrm{i}+1)}} ; q_{n+1}^{i+1}=q_{n+1}^{i}+\lambda \sqrt{2 / 3} \Delta t$. Solve iteratively $\varphi\left(s_{n+1}^{i+1}, p_{n+1}^{i+1}, q_{n+1}^{i+1}\right)=0$ for $\lambda$ withinitial $i=0$, and then update $q_{n+1}$ and $\sigma_{n+1}$. Solve $\boldsymbol{V}_{e(\mathrm{n}+1)}^{(\mathrm{i}+1)} \cdot \boldsymbol{V}_{e(\mathrm{n}+1)}^{(\mathrm{i}+1)}=2 \boldsymbol{B}_{e(\mathrm{n}+1)}^{(\mathrm{i}+1)}+\boldsymbol{I}$ for $\boldsymbol{V}_{e(\mathrm{n}+1)}^{(\mathrm{i}+1)}$.

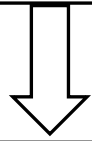

5. Evaluate plastic deformation gradient $\overline{\boldsymbol{F}}_{p(n+1)}$ using

$$
\overline{\boldsymbol{F}}_{p(n+1)}=V_{e(n+1)}^{-1} \cdot F_{n+1} . \text { Save } \boldsymbol{\sigma}_{n+1}, q_{n+1} \text {, and } \overline{\boldsymbol{F}}_{p(n+1)} \text {, and EXIT. }
$$




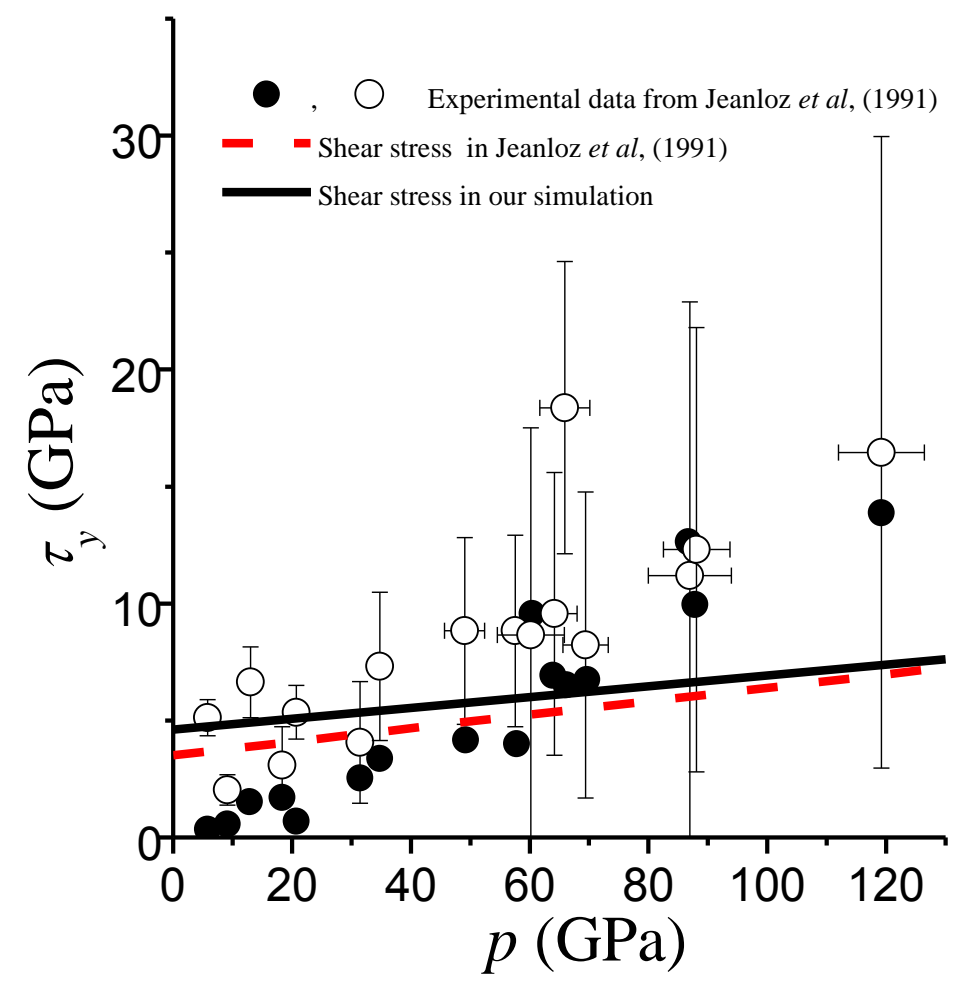

Fig. 1. Shear strength in experiments and approximations in our current simulations and in Jeanloz et al. (1991). Solid symbols without error bar are experimental data calibrated from sample pressure gradient, and open symbols with error bar are experimental data calibrated from diamond anvil measurements. The solid and dashed lines are the approximations of experimental data in our current simulations and in Jeanloz et al. (1991). 


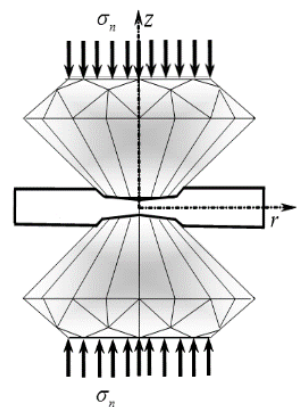

(a)

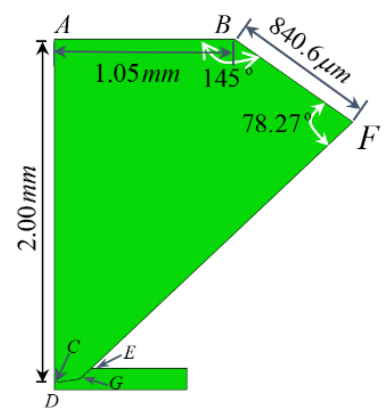

(b)

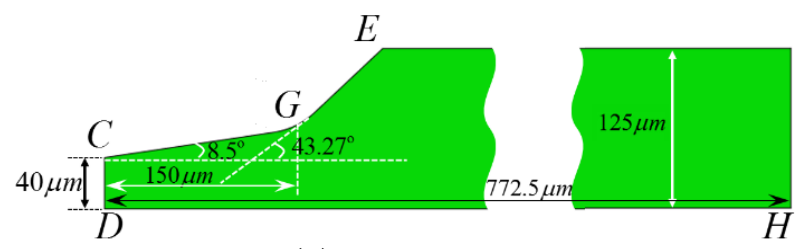

(c)

Fig. 2. (a) Diamond anvil cell scheme, (b) a quarter of the sample and anvil in the initial undeformed state and the geometric parameters of an anvil, and (c) the geometric parameters of a sample. 


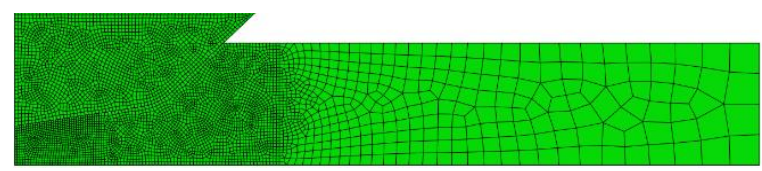

(a)

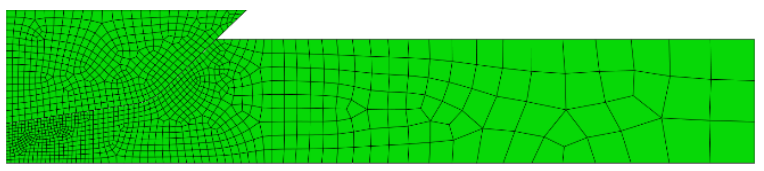

(b)

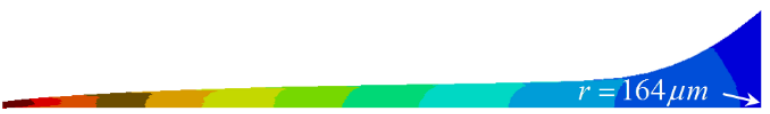

(c) (d)

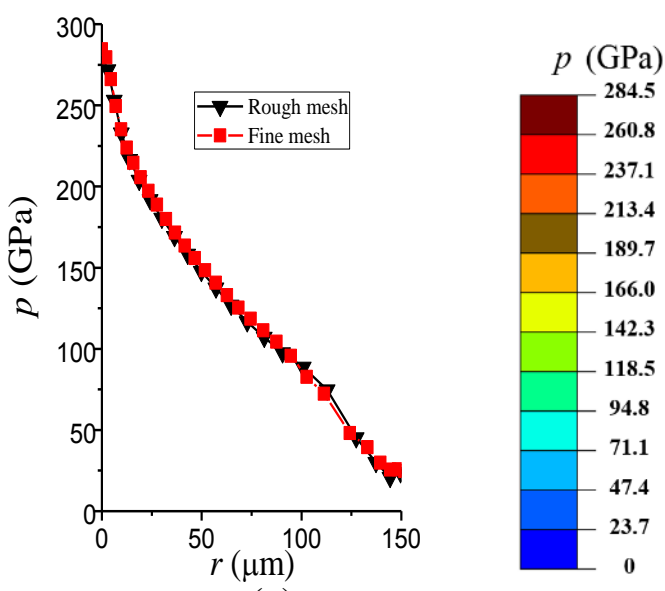

(e)

Fig. 3 Mesh-independence of FEM solutions. (a) Fine mesh with 3198 elements in a sample and (b) rough mesh with 919 elements. Pressure distribution in the sample for fine mesh (c) and rough mesh (d). (e) Pressure distributions at the contact surface for fine and rough meshes. 


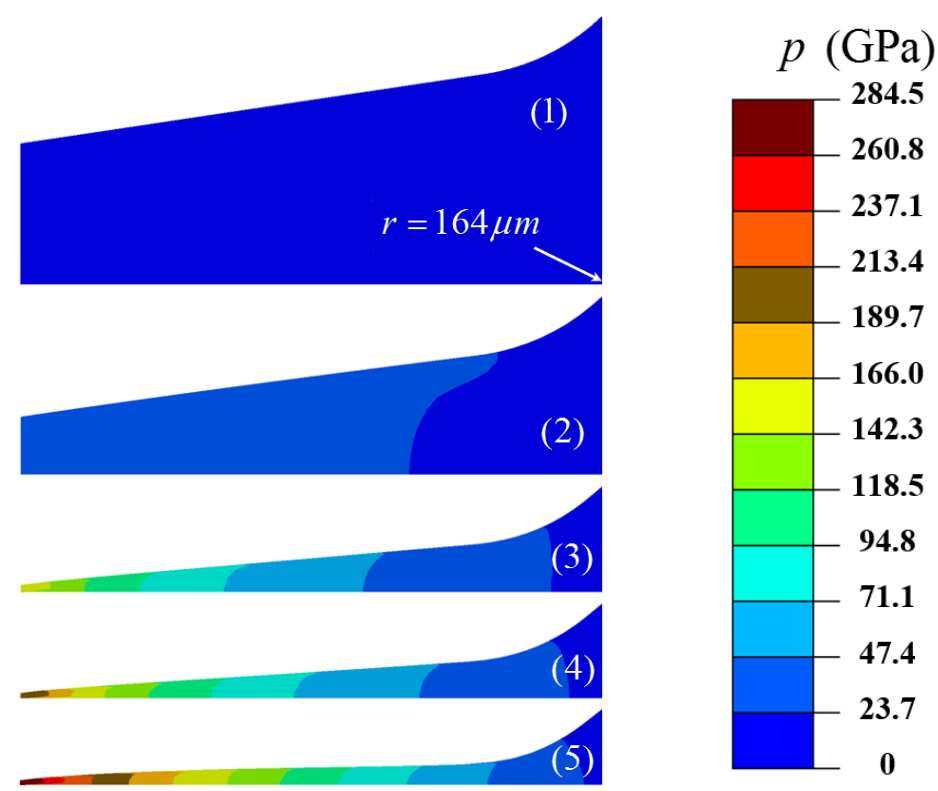

Fig. 4 Pressure distribution in the sample $(0 \leq r \leq 164 \mu \mathrm{m})$ with an increasing applied normal stress $\sigma_{n}$. The normal stress $\sigma_{n}$ is $0(1), 1.022 \mathrm{GPa}(2), 1.652 \mathrm{GPa}(3), 1.903 \mathrm{GPa}(4)$, and $2.546 \mathrm{GPa}(5)$. 


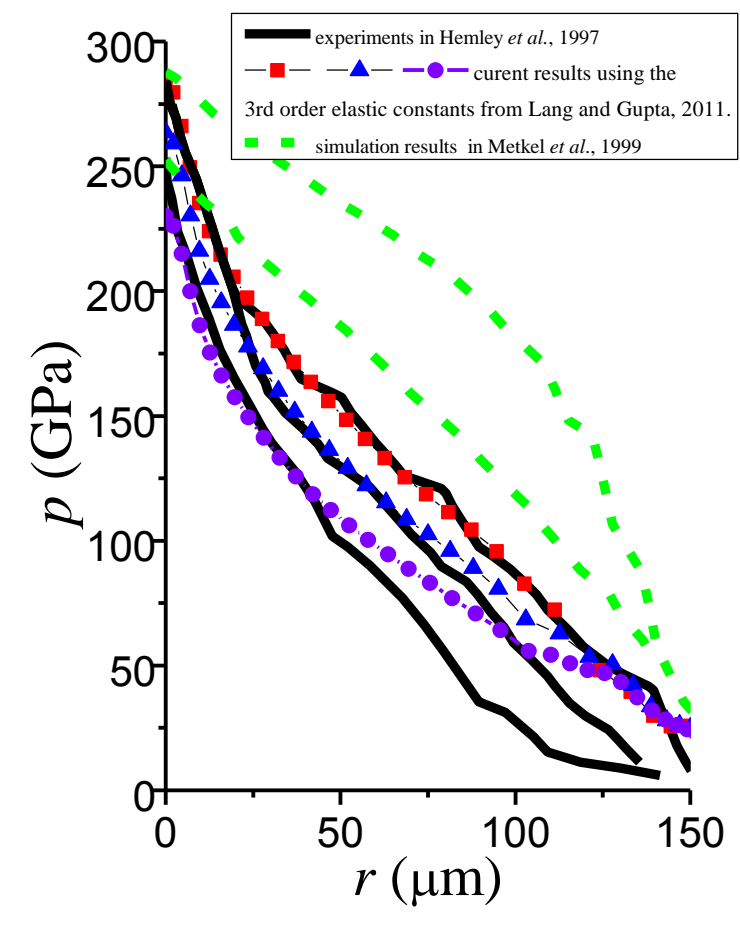

(a)

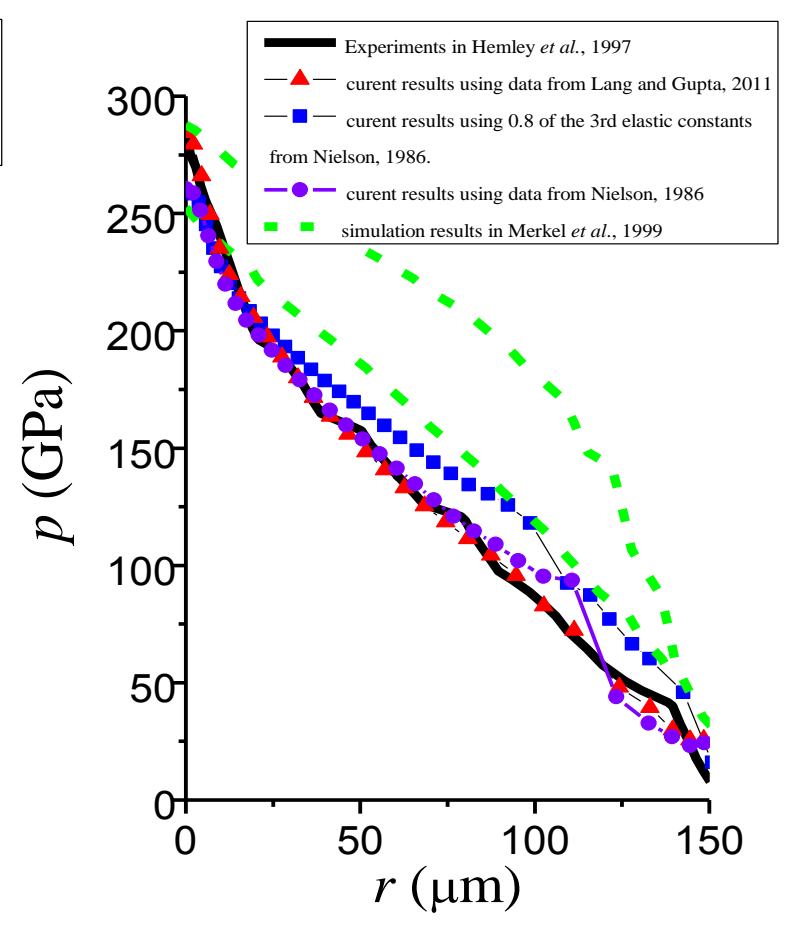

(b)

Fig. 5. Pressure distributions at the contact surface. Lines with symbols are the current simulation results with different third-order elastic constants of diamond, which are taken from Lang and Gupta (2011) in (a) and from Lang and Gupta (2011), Nielsen (1986) and 0.8 of the third-order constants from (Nielsen, 1986) in (b)); black solid lines are experimental data from Hemley et al. (1997); the green dashed line is the simulation results from Merkel et al. (1999). 


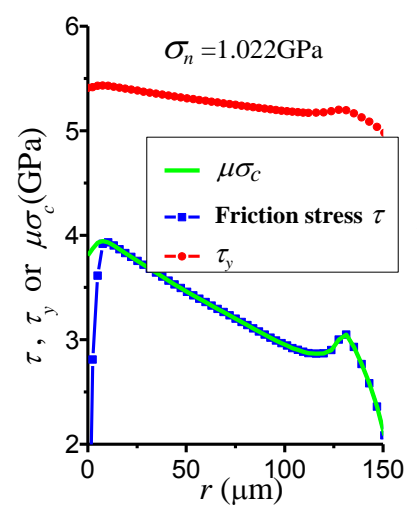

(a)

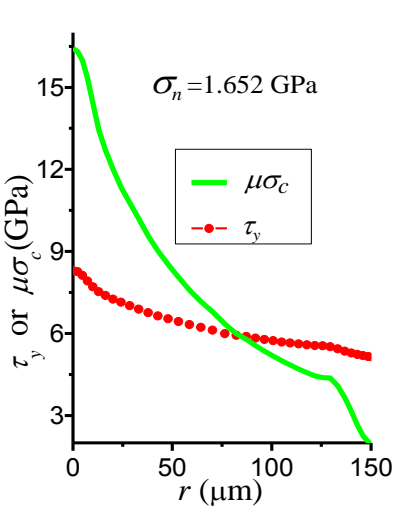

(b)

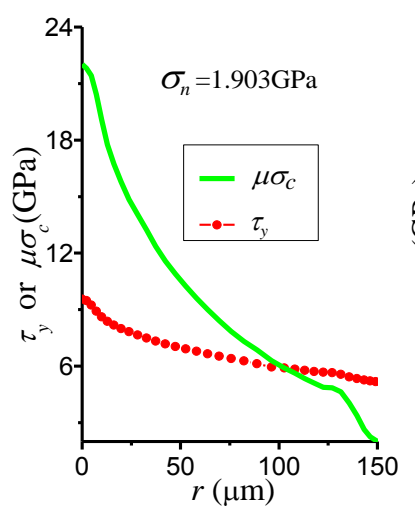

(c)

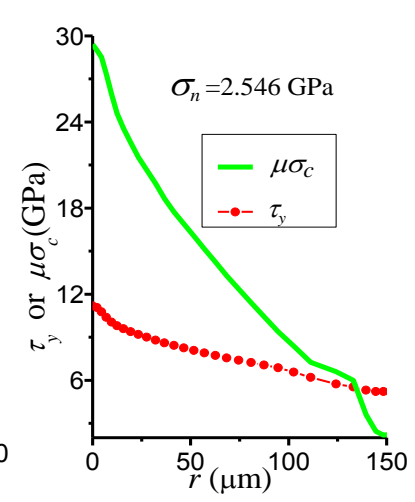

(d)

Fig. 6. Distributions of the shear friction stress $\tau$ in (a), yield strength in shear $\tau_{y}$ and $\mu \sigma_{c}$ at the contact surface at different applied normal stresses $\sigma_{n} . \sigma_{c}$ is the normal contact stress at the contact surface between the diamond and the sample. The shear friction stress in the sliding region $\tau=\min \left(\mu \sigma_{c}, \tau_{y}(p)\right)$, i.e., there is the Coulomb sliding at the periphery and the plastic sliding at the center. In the small cohesion zone (shown for the smallest $\sigma_{n}$ only) $\tau<\min \left(\mu \sigma_{c}, \tau_{y}(p)\right)$. 


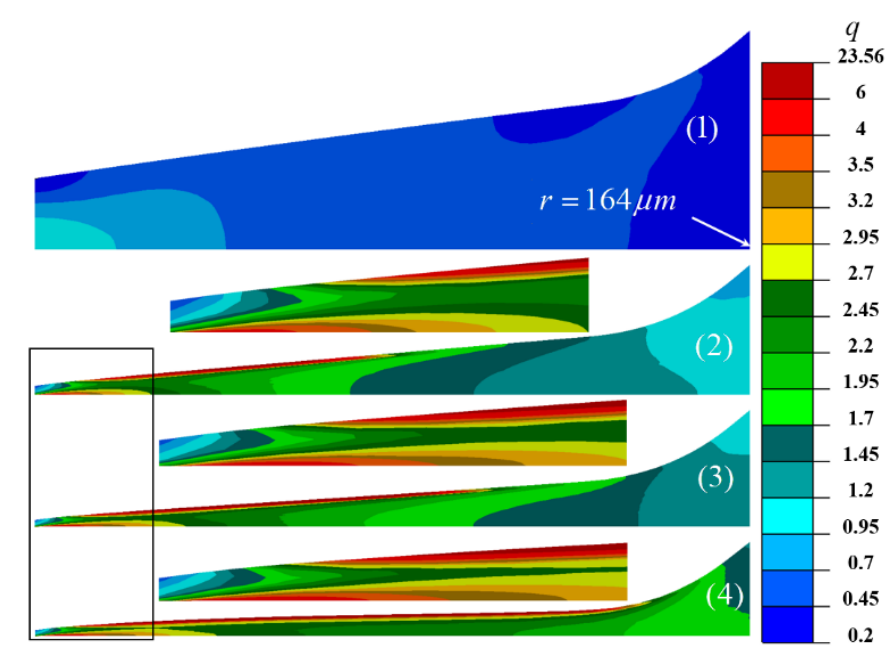

Fig. 7. Distribution of the accumulated plastic strain $q$ in the sample $(0 \leq r \leq 164 \mu \mathrm{m})$ with an increasing applied normal stress $\sigma_{n}: 1.022 \mathrm{GPa}(1), 1.652 \mathrm{GPa}(2), 1.903 \mathrm{GPa}(3)$, and $2.546 \mathrm{GPa}$ (4). The zoomed central part of a sample is shown above the sample for each applied normal stress. 


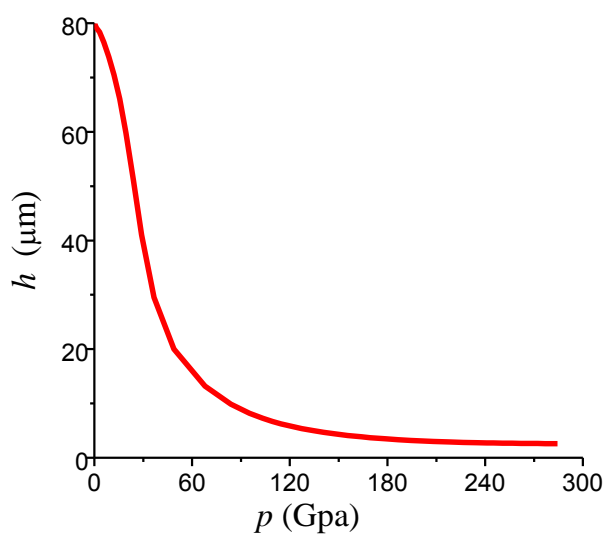

(a)

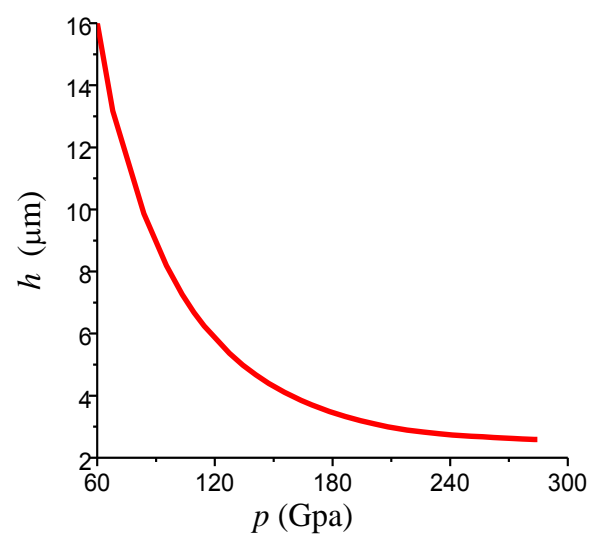

(b)

Fig. 8. The variation of the sample thickness $h$ at the center with growing pressure $p$ at the contact surface at the center. The third-order elastic constants are taken from Lang and Gupta (2011). The curve in (b) is a part of the curve in (a) for the pressure $p>60 \mathrm{GPa}$. 


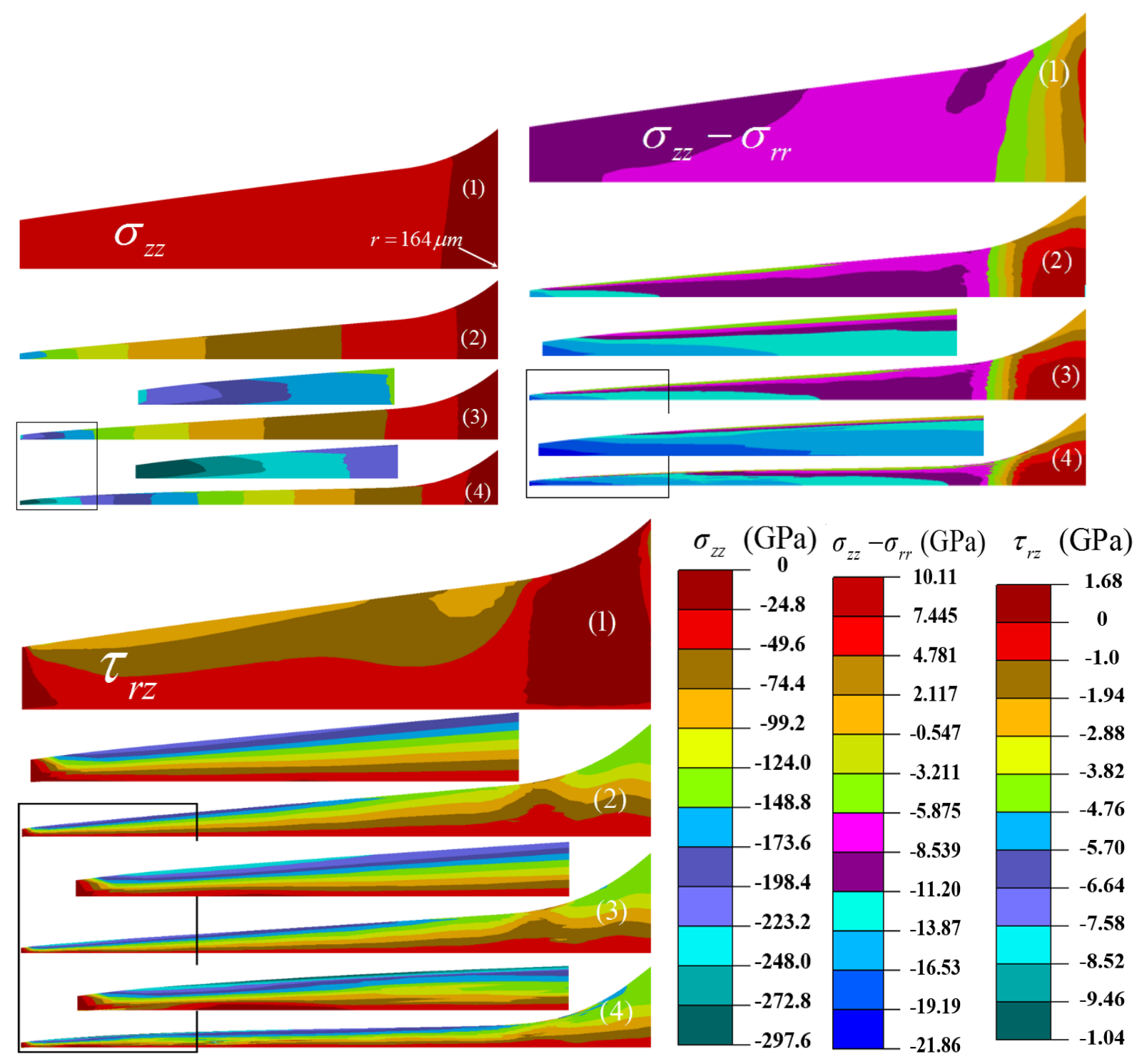

Fig. 9 Distributions of stresses $\sigma_{z z}, \sigma_{z z}-\sigma_{r r}$, and $\tau_{z r}$ in the sample $(0 \leq r \leq 164 \mu \mathrm{m})$ with an increasing applied stresses. The applied normal stress $\sigma_{n}$ at the top surface of the diamond in Fig. 2 (a) is 1.022 GPa (1), $1.652 \mathrm{GPa}(2), 1.903 \mathrm{GPa}$ (3), and $2.546 \mathrm{GPa}$ (4). 


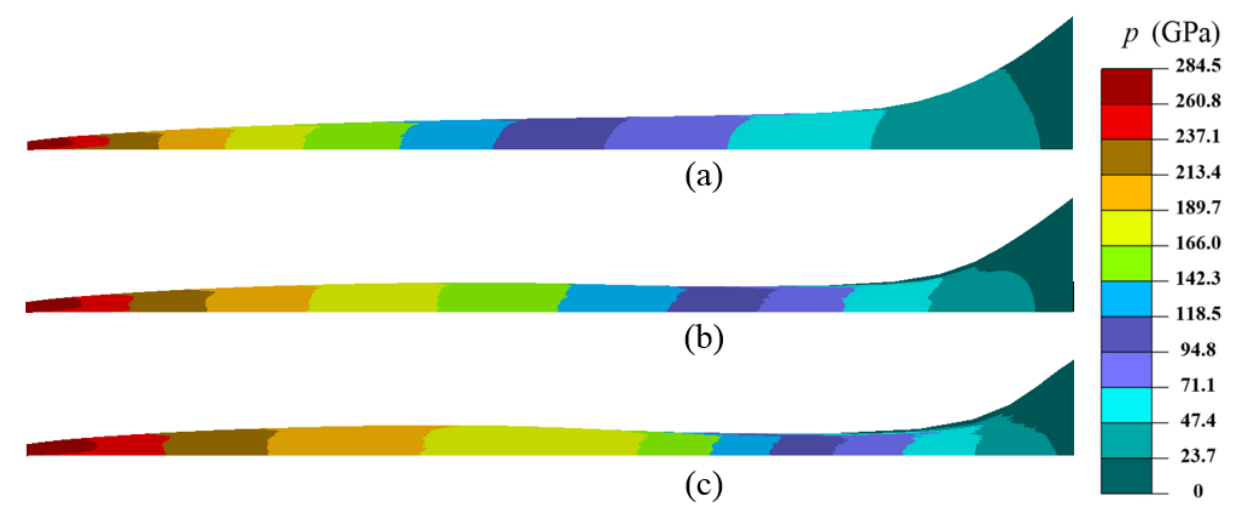

Fig. 10. Pressure distribution in the sample $(0 \leq r \leq 164 \mu \mathrm{m})$ when the pressure in the center reaches $285 \mathrm{GPa}$. The third-order elastic constants are taken from Lang and Gupta (2011) in (a), from Nielsen (1986) in (b), and 0.8 of the third-order constants from (Nielsen, 1986) in (c). The thickness of a sample at the center for (a), (b), and (c) is 2.6, 3.2, and 3.6 $\mu \mathrm{m}$. 


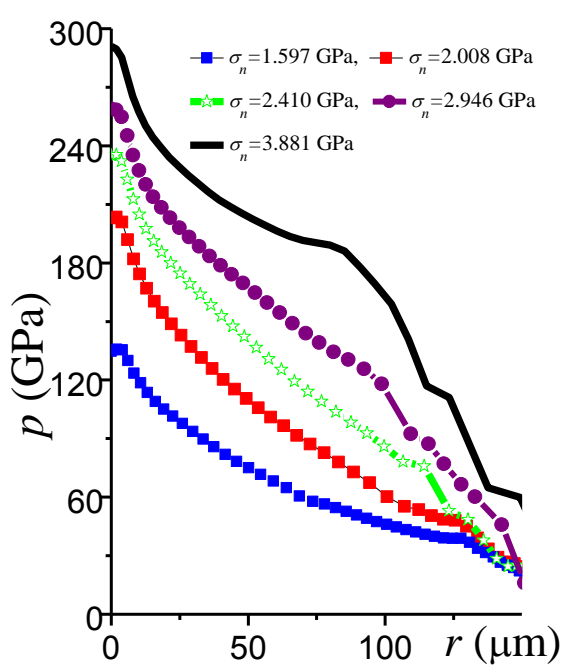

(a)

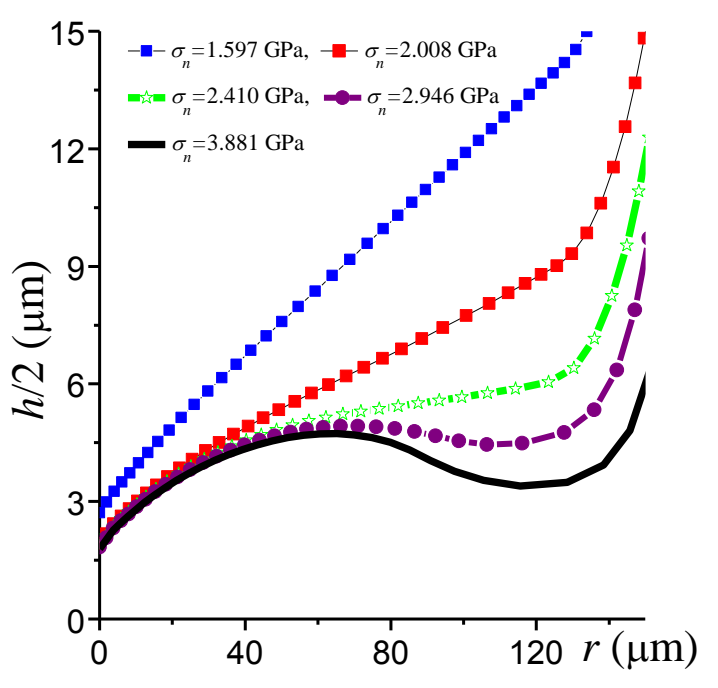

(b)

Fig. 11. Pressure distributions (a) at the contact surface of sample with an increasing applied normal stress $\sigma_{n}$ and half of a thickness of the sample (b) corresponding pressure curves in (a) designated with the same symbols and colors. The third-order elastic constants are taken as 0.8 of the third-order constants from Nielsen (1986). 


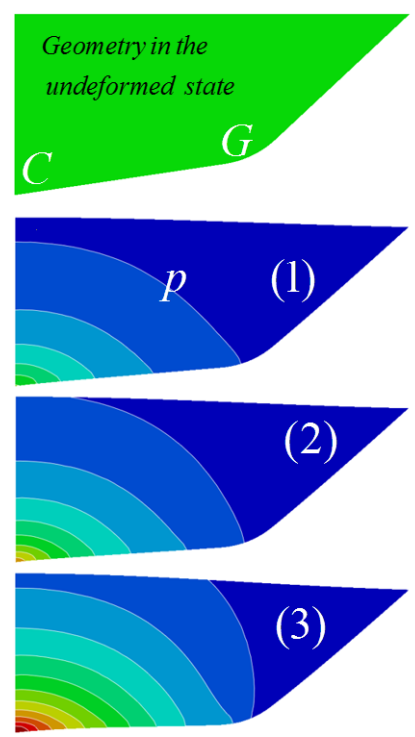

(a)

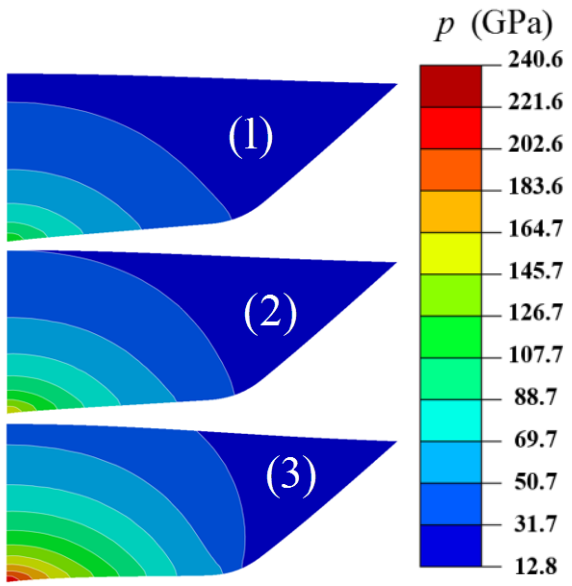

(b)

Fig. 12 Pressure distribution in the anvil with the growth of applied loading obtained with thirdorder elastic constants from Lang and Gupta (2011) (a) and Nielsen (1986) (b). The applied normal stress $\sigma_{n}$ is $1.652 \mathrm{GPa}(1), 1.903 \mathrm{GPa}(2)$, and $2.546 \mathrm{GPa}$ (3). Points $\mathrm{C}$ and $\mathrm{G}$ in the undeformed state (the green figure) corresponds to the Points $\mathrm{C}$ and $\mathrm{G}$ in Fig. 2 (b). 


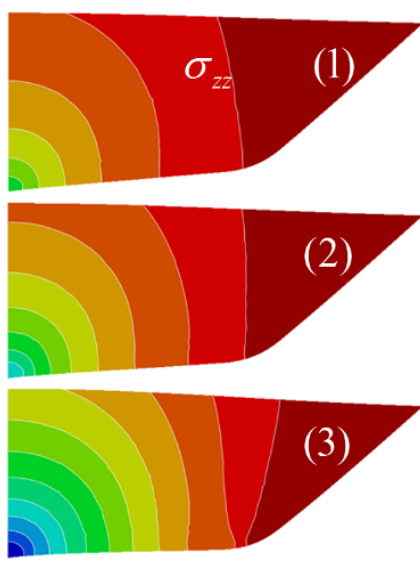

(a)

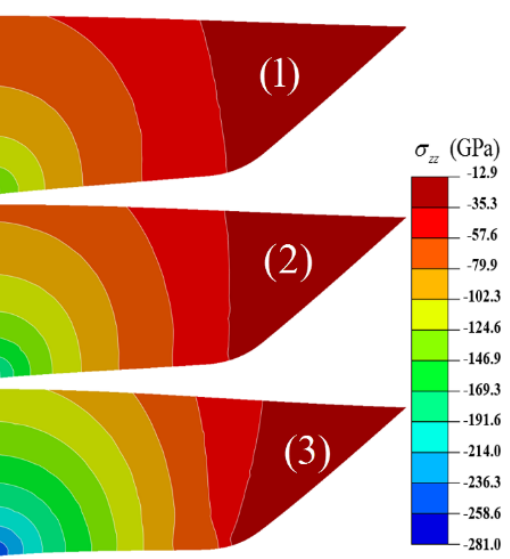

(b)

Fig. 13 Distribution of stress $\sigma_{z z}$ in the anvil with the growth of applied loading obtained with thirdorder elastic constants from Lang and Gupta (2011) (a) and Nielsen (1986) (b). The applied normal stress $\sigma_{n}$ is $1.652 \mathrm{GPa}(1), 1.903 \mathrm{GPa}(2)$, and $2.546 \mathrm{GPa}(3)$. 


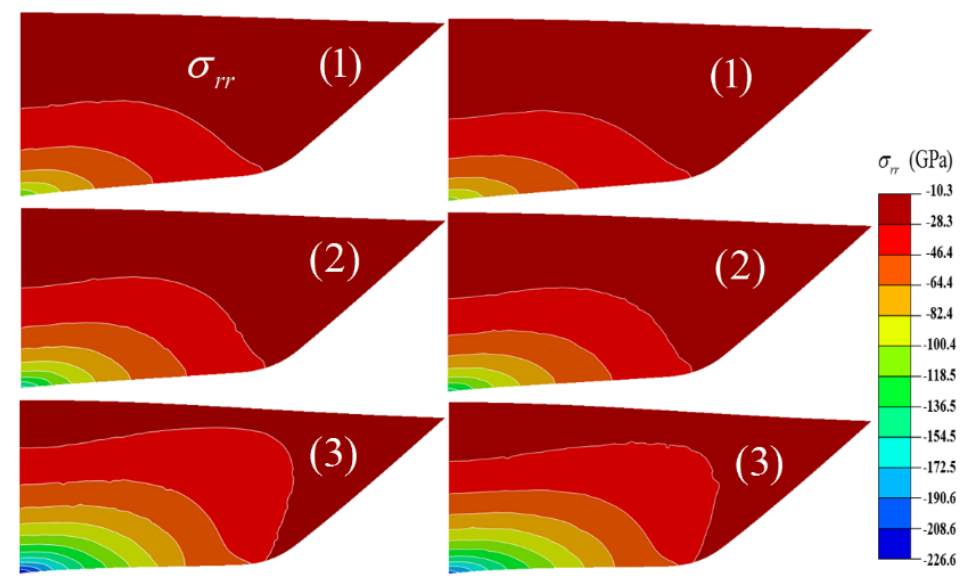

(a)

(b)

Fig. 14 Distribution of stress $\sigma_{r r}$ in the anvil with the growth of applied loading obtained with thirdorder elastic constants from Lang and Gupta (2011) (a) and Nielsen (1986) (b). The applied normal stress $\sigma_{n}$ is $1.652 \mathrm{GPa}(1), 1.903 \mathrm{GPa}(2)$, and $2.546 \mathrm{GPa}$ (3). 


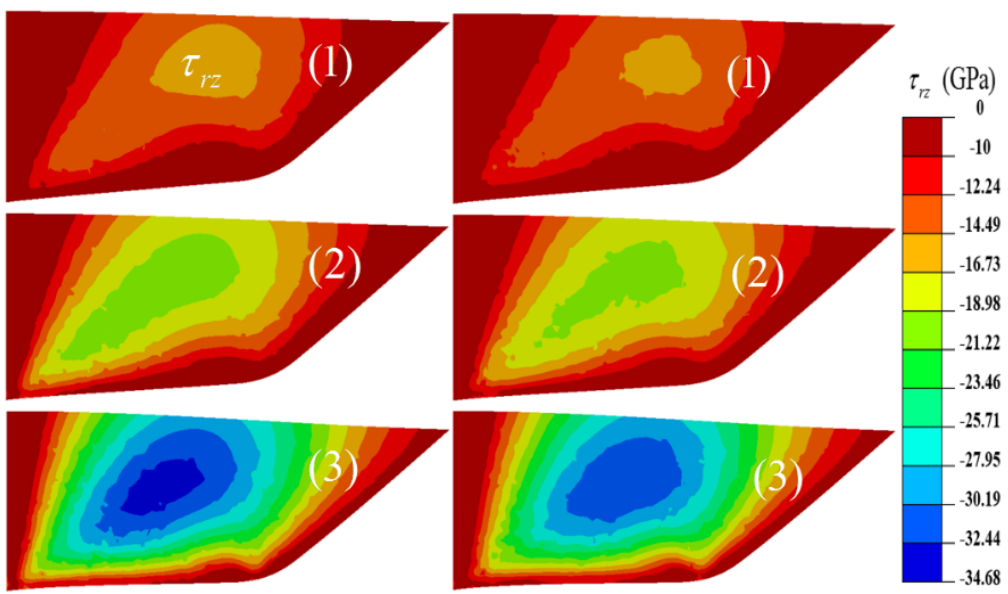

(a)

(b)

Fig. 15. Distribution of shear stress $\tau_{r z}$ in the anvil with the growth of applied loading obtained with the third-order elastic constants from Lang and Gupta (2011) (a) and Nielsen (1986) (b). The applied normal stress $\sigma_{n}$ is $1.652 \mathrm{GPa}(1), 1.903 \mathrm{GPa}(2)$, and $2.546 \mathrm{GPa}$ (3). 


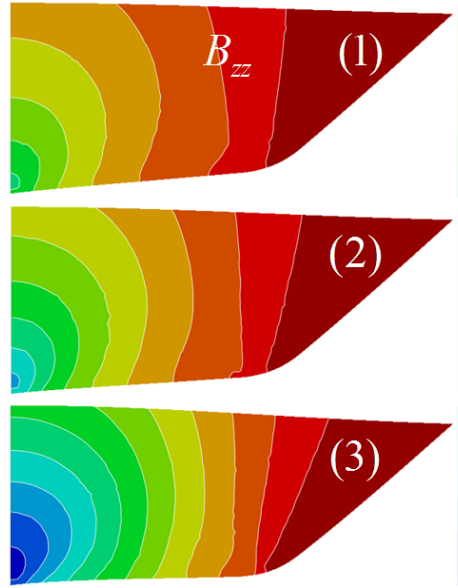

(a)

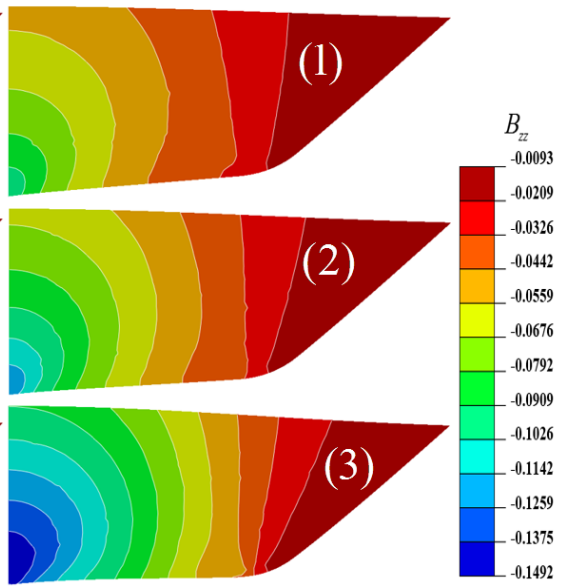

(b)

Fig. 16. Distribution of strain $B_{z z}$ in the anvil with the growth of applied loading obtained with the third-order elastic constants from Lang and Gupta (2011) (a) and Nielsen (1986) (b). The applied normal stress $\sigma_{n}$ is $1.652 \mathrm{GPa}(1), 1.903 \mathrm{GPa}(2)$, and $2.546 \mathrm{GPa}$ (3). 


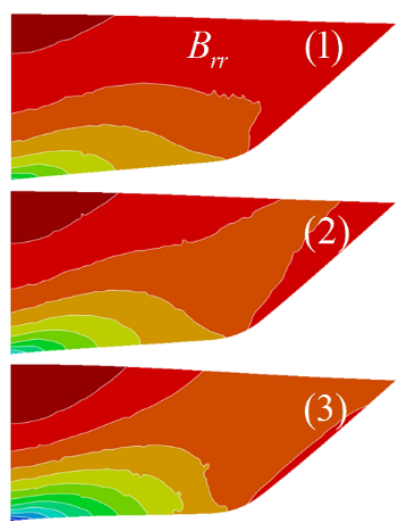

(a)

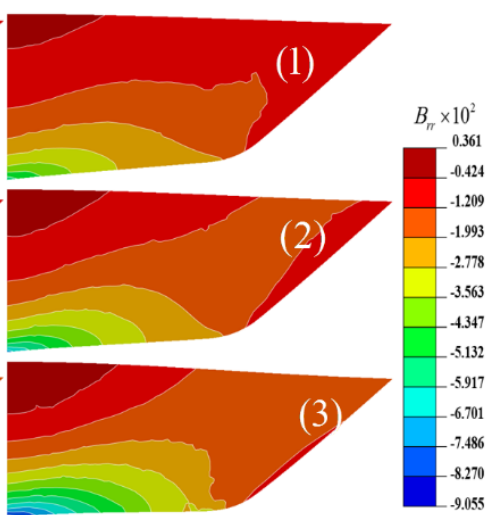

(b)

Fig. 17 Distribution of strain $B_{r r}$ in the anvil with the growth of applied loading obtained with the third-order elastic constants from Lang and Gupta (2011) (a) and Nielsen (1986) (b). The applied normal stress $\sigma_{n}$ is $1.652 \mathrm{GPa}(1), 1.903 \mathrm{GPa}(2)$, and $2.546 \mathrm{GPa}(3)$. 


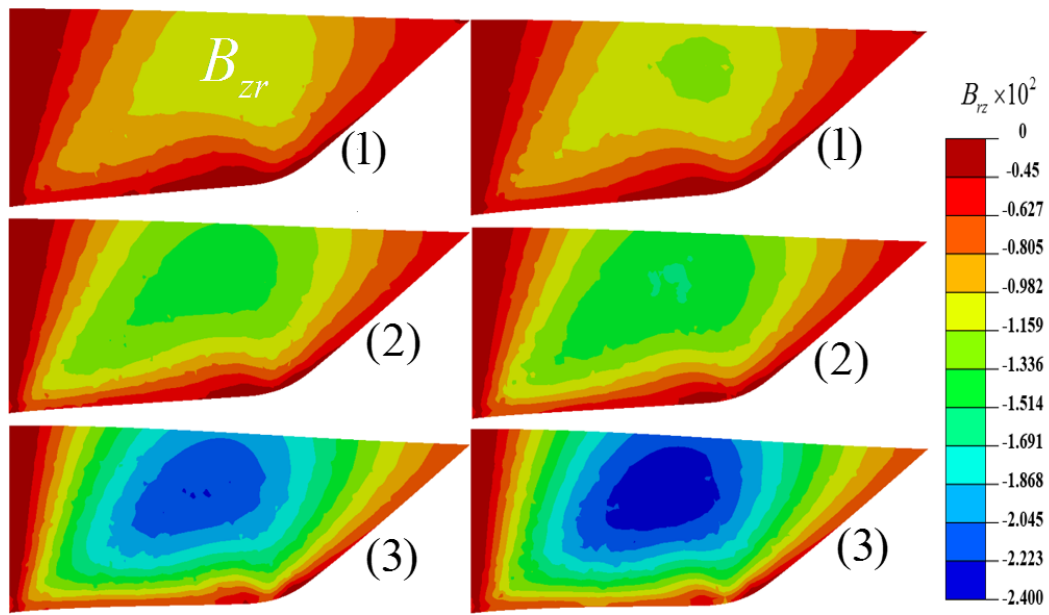

(a)

(b)

Fig. 18 Distribution of shear strain $B_{r z}$ in the anvil with the growth of applied loading obtained with the third-order elastic constants from Lang and Gupta (2011) (a) and Nielsen (1986) (b). The applied normal stress $\sigma_{n}$ is $1.652 \mathrm{GPa}(1), 1.903 \mathrm{GPa}(2)$, and $2.546 \mathrm{GPa}$ (3). 

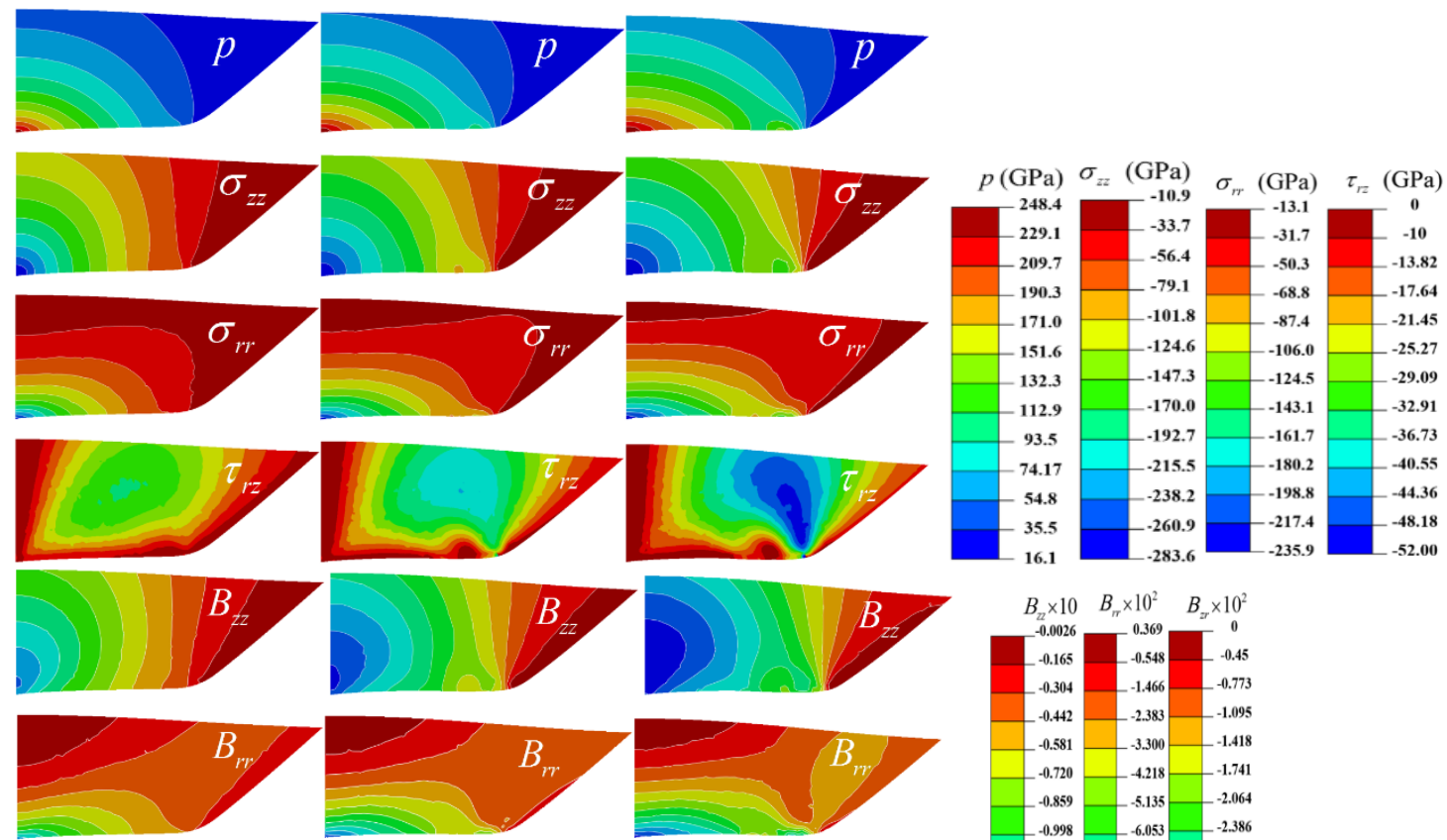

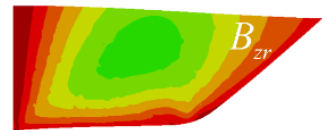

(a)

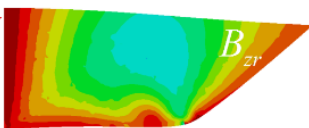

(b)

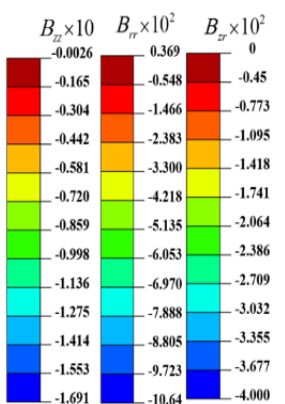

(c)

Fig. 19 Distributions of pressure $p$, stresses, $\sigma_{z z}, \sigma_{r r}$ and $\tau_{z r}$, and strains $B_{z z}, B_{r r}$, and $B_{r z}$ in the anvil when the maximum pressure in the sample reaches $285 \mathrm{GPa}$. Results are obtained with the third-order elastic constants from Lang and Gupta (2011) (a) and Nielsen (1986) (b), and 0.8 of the third-order elastic constants of from Nielsen (1986) (c). The resultant force $F$ is $8818 \mathrm{~N}$ (a), $11061 \mathrm{~N}$ (b), and $13221 \mathrm{~N}$ (c). The applied normal stress $\sigma_{n}$ is $2.546 \mathrm{GPa}(\mathrm{a}), 3.124 \mathrm{GPa}(2)$, and $3.817 \mathrm{GPa}$ (c). 